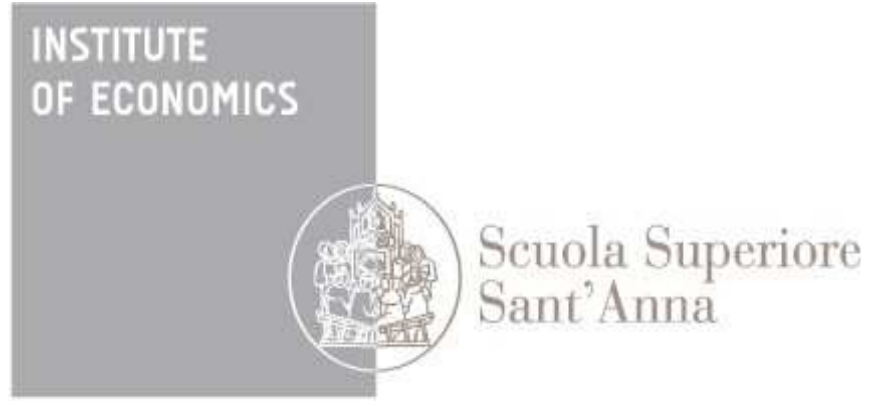

LEM | Laboratory of Economics and Management

Institute of Economics

Scuola Superiore Sant'Anna

Piazza Martiri della Libertà, 33 - 56127 Pisa, Italy ph. +3905088.33 .43$

institute.economics@sssup.it

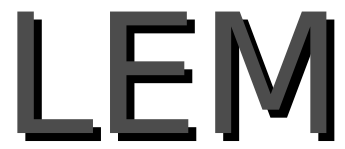

Working Paper Series

\title{
Momentum and Reversal in Financial Markets with Persistent Heterogeneity
}

Giulio Bottazzi ○

Pietro Dindo *

Daniele Giachini ${ }^{\circ}$

'Institute of Economics, Scuola Superiore Sant'Anna, Pisa, Italy

* Dipartimento di Economia, Universita' Ca' Foscari, Venezia, Italy 


\title{
Momentum and Reversal in Financial Markets with Persistent Heterogeneity*
}

\author{
Giulio Bottazzi ${ }^{1}$, Pietro Dindo ${ }^{2,1}$, and Daniele Giachini ${ }^{1}$ \\ ${ }^{1}$ Istituto di Economia, Scuola Superiore Sant'Anna, Piazza Martiri della Libertà 33, \\ 56127 Pisa - Italy \\ 2 Dipartimento di Economia, Università Ca' Foscari Venezia, San Giobbe, Cannaregio \\ 873, 30121 Venezia - Italy
}

February 9, 2018

\begin{abstract}
This paper investigates whether short-term momentum and long-term reversal may emerge from the wealth reallocation process taking place in speculative markets. We assume that there are two classes of investors who trade long-lived assets by holding constantly rebalanced portfolios based on their beliefs. Provided beliefs, and thus portfolios, are sufficiently diversified, all investors survive in the long-run and, due to waves of mispricing, the resulting equilibrium returns exhibit long-term reversal. If, moreover, asset dividends are positively correlated, investors' profitable trades become positively correlated too, thus generating short-term momentum in equilibrium returns. We use the model to replicate the performance of the Winners and Losers portfolios highlighted by the empirical literature and to provide insights on how to improve upon them. Finally, we show that dividend positive autocorrelation is positively related to momentum and negatively related to reversal while diversity of beliefs is positively related to both momentum and reversal.
\end{abstract}

Keywords: Market Efficiency; Heterogeneous Beliefs; Speculation; Short-term Momentum; Long-term Reversal.

JEL Classification: C60, D53, G02, G12, G14

\footnotetext{
*Pietro Dindo has been supported by the Marie Curie International Outgoing Fellowship PIOF-GA-2011300637 within the 7th European Community Framework Programme. Daniele Giachini gratefully acknowledges the hospitality of the Department of Banking and Finance at the University of Zurich. This research has received funding from the European Union's Horizon 2020 research and innovation programme under grant agreement No 640772 - DOLFINS.
} 


\section{Introduction}

The cornerstone of the Efficient Market Hypothesis is the martingale property: properly discounted and risk adjusted asset returns are equal, in expectation, to the risk free rate, no matter the information set on which they are conditioned. However, in the last decades, several empirical studies showed how two peculiar patterns seem to be quite common: short-term momentum and long-term reversal. According to the former, excess returns tend to be positive (negative) after positive (negative) excess returns over recent lags, while according to the latter, they tend to be negative (positive) after positive (negative) excess returns over distant lags. ${ }^{1}$

In this paper, we explore whether the two phenomena may be due to the wealth reallocation process that takes place in speculative markets. In particular, we assume that agents invest in long-lived assets using constantly rebalanced portfolios derived in accordance with their beliefs. Due to beliefs heterogeneity, agents take different positions and thus gain or lose wealth depending on dividend payments. The ensuing wealth dynamics amplify price movements, generating positive capital gains after high dividends and negative otherwise. We investigate whether such return dynamics can be responsible for short-run momentum and long-run reversal in equilibrium. We also investigate the existence and nature of profitable portfolio strategies and how momentum and reversal react to variations in the parameters of the economy, such as beliefs dispersion and dividend autocorrelation.

On the modeling side, we make a number of choices to simplify the set-up and, at the same time, have an asset price process that is the Walrasian equilibrium outcome of a financial market with heterogeneous traders. We consider an endowment economy in discrete time with two possible states of the world in every period and an infinite horizon. The aggregate endowment is spanned by two long-lived risky assets and each asset pays the dividend only in one of the two states. A key assumption is that dividends, and thus states, follow a Markov process where the probability that an asset pays dividends is higher if the same asset payed dividends in the last period. Asset prices are determined in equilibrium in a competitive market with two (classes of) agents. Portfolio and saving decisions are kept simple. Agents' saving rate is constant and homogeneous while demands in assets are derived as constantly rebalanced portfolios. These portfolios are derived in accordance to each agent's beliefs as Subjectively Generalized Kelly portfolios (Evstigneev et al., 2008; Bottazzi et al., 2017), or "bet your beliefs" as in Kelly (1956) and Breiman (1961) $:^{2}$

\footnotetext{
${ }^{1}$ See Section 2 for an account of the related literature.

${ }^{2}$ As we shall make clear in Section 4, these portfolio and saving rules correspond to those of an agent who maximizes the expected discounted stream of consumption when her beliefs get influenced by market prices, see Bottazzi et al. (2017), or the related work on the Fractional Kelly rule in heterogeneous agent economies Kets et al. (2014), Bottazzi and Giachini (2016),
} 
Beliefs are assumed to be constant (agents think that the state process is i.i.d.) and biased enough to sustain long-run heterogeneity. This is a behavioral assumption consistent with the view that agents use trading rules that do not reflect all the complexity of reality (see e.g. Gigerenzer et al., 1999; Gigerenzer and Brighton, 2009; Gigerenzer and Gaissmaier, 2011). In the words of Daniel and Titman (1999): "individuals have limited processing ability, and hence use vague, ad hoc rules to translate the information they receive into estimates of cash flows and firm valuations".

In this setting, we are able to derive the explicit stochastic difference equation underlying the price process and completely characterize asset conditional excess returns. As a main result we show that the reallocation of wealth generated by market selection forces, together with the underlying Markov process and belief heterogeneity, is responsible for both short-term momentum and long-term reversal. The effects occur even if agents do not revise their beliefs when a particular state comes out. In fact, the fundamental mechanisms that underlies our results is the interplay between two forces: the positive autocorrelation in the dividend process, which triggers momentum, and the mispricing that may occur under belief heterogeneity, causing return reversal. While the former effect is constant, the latter increases when several states of the same type appear. In the short-run, the positive autocorrelation overwhelms the mispricing and the result is shortterm momentum. Conversely, when a long sequence of high dividend payments is realized, the mispricing becomes the strongest force and long-term reversal is observed.

Our analysis has several implications. First, we show how, conditioning on past returns, it is possible to replicate the superior empirical performance of the Winners portfolio over the Losers portfolio in the short-run and of the Losers portfolio over the Winners portfolio in the long-run 3 Second, we also show how to use the joint information of returns and prices to improve upon these portfolios and build short-selling constrained portfolios that deliver a positive expected return in every period. Third, we prove that, removing the short-selling constraint, it is possible to build a zero-cost portfolio that provide a positive expected payoff in every period 4 Fourth, we find that increasing the dispersion of beliefs affects positively both short-run momentum and long-run reversal. Fifth, increasing the strength of dividends positive autocorrelation affects positively short-run momentum and negatively long-run reversal. Those findings, which have been in part confirmed by empirical studies, can provide new hints for future empirical research.

The paper is organized as follows. In Section 2, we review the related literature.

Bottazzi and Giachini (2017), and Dindo and Massari (2017).

${ }^{3}$ See section 2 for more details on empirical results concerning Winners-Losers portfolios.

${ }^{4}$ In doing so, we neglect the price impact of such portfolio strategies. Their inclusion in the set of actually trading strategies is in the scope of future works. 
In Section 3, we introduce the formal definitions of momentum and reversal used throughout our analysis. In Section 4, we present our formal model. In Section 5. we investigate how and under which conditions the model produces momentum and reversal. In Section 6, we study the implications of our model. Section 7 concludes.

\section{Literature Review}

Several empirical studies have investigated momentum and reversal in asset returns, here we briefly report those that are most significant for our analysis. The earliest empirical research on the topic focuses on observing the returns of portfolios built considering stocks with particularly good or bad performances over previous periods. De Bondt and Thaler (1985, 1987) consider two portfolios: the Losers portfolio, made by stocks with poor performances over the previous period (36-60 months), and the Winners portfolio, made by stocks with high performances over the same period. They show that Losers portfolio systematically outperforms Winners portfolio over the following 36-60 months. Jegadeesh and Titman (1993) find that strategies which buy stocks that have showed good performances in the past 3-12 months and sell stocks that have, instead, showed poor performances in the past 3-12 months generate positive returns in the following 3-12 months.

Relying on similar techniques, several contributions enriched and complemented those findings. In particular, Bernard and Thomas (1989) and Bernard (1992) find that, depending on observable public events, stocks abnormal returns tend to move in the same direction as the initial event and to keep the trend for the following 60-180 trading days. Chopra et al. (1992) confirm the results of De Bondt and Thaler, showing also how the mean-reverting behavior is distinct from tax-loss selling effects and that it is stronger for smaller firms than for larger firms. Chan et al. (1996) show how both the most recent earning surprise and the past six-month return are able to predict drift in future returns. Daniel and Titman (1999) find that momentum effects are stronger for growth stocks than value stocks. Moskowitz and Grinblatt (1999) find evidence of momentum in portfolios built at the industry level. Hong et al. (2000) show that momentum profits are negatively correlated to firm size and to analyst coverage. Jegadeesh and Titman (2001) confirm their previous findings and also find evidence for return reversal from 48 to 60 months after the portfolio formation. Verardo (2009) finds that momentum profits are larger for those portfolios composed by stocks characterized by higher belief heterogeneity, using dispersion of analyst forecasts as proxy. Asness et al. (2013) find evidence for momentum profits across eight diverse markets and asset classes, expanding the previous results of Rouwenhorst (1998).

As a general criticism with respect to approaches based on portfolio compari- 
son, Lo and MacKinlay (1990) and Lewellen (2002) point out that the return of a portfolio built considering previous performances can be particularly high (or low) because returns are autocorrelated, or a form of cross-serial correlation among the securities that form the portfolio exists (lead-lag effect). Indeed Lewellen (2002) shows that the negative cross-correlation among assets may account for a significant share of momentum profits. At the same time, other empirical investigations have explored the time-series dimension rather than the portfolio dimension. Moskowitz et al. (2012) find strong "time series momentum" (autocorrelation in returns) that partially reverts in the long-run for several future and forward contracts. Balvers et al. (2000), Gropp (2004), and Mukherji (2011) find evidence of mean reverting behavior in return time-series over lags of 12-60 months. Mukherji also suggests that this effect have weakened in recent years for large firms, while it persists for small firms. All these contributions confirm and extend the previous findings of Poterba and Summers (1988), Jegadeesh (1990) and Cutler et al. (1991) about positive autocorrelation of returns between 1 and 12 months and negative autocorrelation between 13 and 24 months.

The theoretical explanations of momentum and reversal mainly belong to the behavioral finance literature, that links the former to under-reaction and the latter to over-reaction of markets to news, caused by investors cognitive biases. Barberis et al. (1998) assume that when news arrive in the market, the representative agent under-reacts, i.e. slowly revises its expectations, so that the price gradually adapts to the new fundamental. This slow adaptation is identified with shortterm momentum. At the same time, when a long enough sequence of news of the same type (either good or bad) occurs, the agent overreacts, i.e. becomes increasingly optimistic or pessimistic, so that prices "overshoot" their fundamental value. This tendency is corrected in the long-run, generating what is recognized as long-term reversal. The particular way in which their representative agent changes its beliefs is justified by means of conservatism and representativeness biases. Such underlying story is in part challenged by Daniel et al. (1998) and Hong and Stein (1999). Daniel et al. (1998), still employing a representative agent model but different cognitive biases (overconfidence and biased self-attribution) and two types of news (private and public), show that short-term momentum can be generated also by agents' continuing over-reaction. Hong and Stein (1999) instead identify in the slow circulation of news among news-watcher traders the roots of under-reaction and the following short-term momentum, while long-term reversal is caused by the action of a different class of traders (who overreact making the price overshoot) coupled with the reaction of the news-watchers (that makes the price go back to the fundamental). A following paper by the same authors (Hong and Stein, 2007) predicts that disagreement (hence heterogeneity of beliefs) not only leads to momentum profits, but the magnitude of those profits is positively 
related to the dispersion of opinions.

Differently from the behavioral finance explanation of momentum and reversal, in our model beliefs are not revised when news arrive in the market. By focusing on the wealth reallocation process that takes place in speculative markets, our analysis highlights a complementary source of momentum and reversal that may reinforce the effects of investors under- and over-reaction. Other works have investigated the role of competitive markets in selecting among trading strategies, providing a theoretical test of the Market Selection Hypothesis of Friedman (1953), at least for financial markets. The seminal contribution in the area is Blume and Easley (1992). Our model, by analyzing a discrete time infinite horizon economy populated by agents with heterogeneous rebalanced portfolios of long-lived assets, is close to Evstigneev et al. (2008) and Bottazzi et al. (2017). Other contributions in the literature considers the outcome of market selection with endogenous portfolios both in discrete time (Sandroni, 2000; Blume and Easley, 2006; Jouini and Napp, 2006) and in continuous time (Jouini and Napp, 2007; Yan, 2008; Cvitanić et al., 2012). Within this Market Selection literature, other models achieve long-run heterogeneity as a result of incomplete markets (Blume and Easley, 2006; Beker and Chattopadhyay, 2010; Coury and Sciubba, 2012), non-tradable labor income (Cogley et al., 2013; Cao, 2017), ambiguity aversion (Condie, 2008; Guerdjikova and Sciubba, 2015), asymmetric and costly information (Sciubba, 2005), non-converging learning (Sandroni, 2005, Beker and Espino, 2011), recursive preferences (Borovička, 2015; Dindo, 2015), solvency constraints (Beker and Espino, 2015). In particular, Beker and Espino (2015) highlight the role that belief heterogeneity and limited enforceability may play for generating short-term momentum and long-term reversal.

\section{Excess Returns, Momentum and Reversal}

As illustrated in the literature review, the definition and investigation of momentum and reversal has taken significantly different forms. Here, we introduce the formal definition based on the time-series dimension of asset returns that we shall use in our analysis.

Consider a long-lived asset (a stock) traded in period $t \in \mathbb{N}$ in a competitive financial market. Call $P_{t}$ its date- $t$ price and $D_{t}$ its date- $t$ dividend. In Section 4 , we shall derive the price process $\left(P_{t}\right)_{t \in \mathbb{N}}$ in equilibrium, starting from agents' asset demands and imposing market clearing. For the moment it is enough to consider it together with the information filtration $\left(\mathcal{J}_{t}\right)_{t \in \mathbb{N}}$ it generates and the related probability triple with measure $\mathrm{P}$. The excess return between dates $t$ and $t+1$ 
reads

$$
r_{t+1}=\frac{P_{t+1}+D_{t+1}-P_{t}}{P_{t}}-r_{f, t},
$$

where $r_{f, t}$ is the risk-free rate. Since all returns will be computed in excess of the risk-free rate, we may drop the adjective excess in what follows.

In benchmark asset pricing models, the equilibrium price process is "fair", that is, conditional on any event which contains information up to $t$, expected returns are zero: 5

$$
E\left[r_{t+1} \mid I_{t}\right]=0 \text { for all } I_{t} \in \mathcal{J}_{t} .
$$

Eq. (1) can fail in many ways. We shall say that the asset price process exhibits momentum when, after a history of returns with a given sign, the expected return has the same sign. On the contrary, a price process exhibits reversal when, after a history returns with a given sign, the expected return has the opposite sign.

To formalize momentum and reversal, we define elements of $\mathcal{J}_{t}$ with returns of a given sign. Given what is known at time $t$, for every $j \in\{1,2, \ldots, t\}$, we define the set of events with last $j$ returns all positive:

$$
\mathcal{R}_{t}^{+}(j)=\left\{r_{t}>0, r_{t-1}>0, \ldots, r_{t-j+1}>0\right\} \in \mathcal{J}_{t} .
$$

Likewise, when the last $j$ returns are all negative we have the set

$$
\mathcal{R}_{t}^{-}(j)=\left\{r_{t}<0, r_{t-1}<0, \ldots, r_{t-j+1}<0\right\} \in \mathcal{J}_{t} .
$$

Since we can have either positive or negative returns, and the length of returns with the same sign can vary from 1 to $j$, we need to distinguish momentum and reversal for all these cases. We start with the following,

Definition 3.1. The asset prices process $\left(P_{t}\right)$ exhibits momentum of order $j$ in $t$ after a history of positive returns and conditionally on the price $P_{t-j+1}$ if

$$
\mathrm{E}\left[r_{t+1} \mid \mathcal{R}_{t}^{+}(j), P_{t-j+1}\right]>0 .
$$

Likewise, $\left(P_{t}\right)$ exhibits momentum of order $j$ in $t$ after a history of negative returns and conditionally on the price $P_{t-j+1}$ if

$$
\mathrm{E}\left[r_{t+1} \mid \mathcal{R}_{t}^{-}(j), P_{t-j+1}\right]<0 .
$$

The asset prices process $\left(P_{t}\right)$ exhibits reversal of order $j$ in $t$ after a history of positive returns and conditionally on the price $P_{t-j+1}$ if

$$
\mathrm{E}\left[r_{t+1} \mid \mathcal{R}_{t}^{+}(j), P_{t-j+1}\right]<0 .
$$

\footnotetext{
${ }^{5}$ We shall show that this is the case in Section 4.1 . We anticipate that having no aggregate risk is crucial for the result, otherwise returns should be risk adjusted.
} 
Likewise, $\left(P_{t}\right)$ exhibits reversal of order $j$ in $t$ after a history of negative returns and conditionally on the price $P_{t-j+1}$ if

$$
\mathrm{E}\left[r_{t+1} \mid \mathcal{R}_{t}^{-}(j), P_{t-j+1}\right]>0 .
$$

Definition 3.1 distinguishes between positive and negative returns and allows the effects to depend on the initial price level. Throughout the analysis, it is convenient to analyze both momentum and reversal using a measure that gets rid of the reference to the sign of previous returns. We do this by averaging with respect to histories with positive or negative returns ${ }^{6}$

Definition 3.2. Let

$$
\phi_{t}\left(j, P_{t-j+1}\right)=\mathrm{E}\left[\operatorname{sgn}\left(r_{t}\right) r_{t+1} \mid \mathcal{R}_{t}^{+}(j) \cup \mathcal{R}_{t}^{-}(j), P_{t-j+1}\right] .
$$

The price process $\left(P_{t}\right)$ exhibits momentum of order $j$ in $t$, given a price $P_{t-j+1}$, if

$$
\phi_{t}\left(j, P_{t-j+1}\right)>0 .
$$

The price process $\left(P_{t}\right)$ exhibits reversal of order $j$ in $t$, given a price $P_{t-j+1}$, if

$$
\phi_{t}\left(j, P_{t-j+1}\right)<0 \text {. }
$$

It might be useful to eliminate the reference to the initial price level and to have a definition of momentum and reversal that depends only on $t$ and $j$, the number of previous periods with returns having the same sign.

Definition 3.3. Fixing $t$ and $j \leq t$ let

$$
\Phi_{t}(j)=\int \phi_{t}(j, P) d F_{t-j+1}(P)=\mathrm{E}\left[\operatorname{sgn}\left(r_{t}\right) r_{t+1} \mid \mathcal{R}_{t}^{+}(j) \cup \mathcal{R}_{t}^{-}(j)\right],
$$

where $F_{t}(P)$ is the cumulative distribution function of $P_{t}$.

The price process $\left(P_{t}\right)$ exhibits momentum of order $j$ in $t$, if

$$
\Phi_{t}(j)>0
$$

The price process $\left(P_{t}\right)$ exhibits reversal of order $j$ in $t$, if

$$
\Phi_{t}(j)<0
$$

\footnotetext{
${ }^{6}$ A similar aggregation is performed in the behavioral finance literature, see e.g. Barberis
} et al. (1998). 
For convenience, we also define

$$
\phi_{\infty}\left(P_{t-j+1}\right)=\lim _{j \rightarrow \infty} \phi_{t}\left(j, P_{t-j+1}\right) \quad \text { and } \quad \Phi_{\infty}=\lim _{j \rightarrow \infty} \Phi_{t}(j) .
$$

In what follows we shall build a simple asset market model where, under dividend positive autocorrelation and long-run heterogeneity triggered by persistent disagreement, equilibrium prices exhibits momentum for low $j \mathrm{~s}$ and reversal for high $j$ s.

\section{The Model}

In this section, we introduce a simple model that allows for the investigation of momentum and reversal in a financial market for long-lived assets with heterogeneous agents. The key element of our model is the wealth reallocation process that takes place in speculative markets. Thus our model belongs to the market selection literature stemming from Blume and Easley (1992). By analyzing a discrete time infinite horizon economy populated by agents with heterogeneous rebalanced portfolios of risky assets, our model is close to Evstigneev et al. (2008) and Bottazzi et al. (2017).

In a nutshell, we consider an endowment economy with two agents whose aggregate endowment is spanned by two long-lived assets. Agents trade assets using simple rules, i.e. constantly rebalanced portfolios, derived in accordance with their beliefs. Due to belief heterogeneity, agents' positions are speculative.

First, we set-up the model and derive the equilibrium asset price process $\left(P_{t}\right)$ as a function of agents' portfolio and saving rules. Next, we characterize the properties of the prices process under different assumptions on agents' behaviors. In Section 5, we use the model to provide conditions for the occurrence of both short-run momentum and long-run reversal, according to the formal definitions of Section 3 ,

Time is discrete and in each date $t \in \mathbb{N}$ one of the two possible states of the world occurs, $s_{t} \in S=\{1,2\} . s^{t}=\left\{s_{1}, \ldots, s_{t}\right\}$ denotes a partial history of states till $t$ and $\Sigma$ is the space of infinite paths of states $\sigma$. $\left(\mathcal{F}_{t}\right)$ is the natural filtration and $\mathcal{F}$ is the $\sigma$-algebra generated by this filtration. All variables that we shall introduce -asset demands, prices, dividends- are adapted to the information filtration $\left(\mathcal{F}_{t}\right)$. In order to ease the notation, their dependence on $\sigma$, and thus on partial histories $s^{t}$, is often dropped.

The economy is populated by $N=2$ (classes of) agents whose aggregate endowment in $t \in \mathbb{N}_{0}=\mathbb{N} \cup\{0\}, Y_{t}$, is measured in units of the consumption good, the numéraire. The aggregate endowment is constant, so that there is no aggregate 
risk, and without loss of generality we set $Y_{t}=1$ for all $t \in \mathbb{N}_{0}$. The aggregate endowment is spanned by $K=2$ long-lived risky assets. Both assets are in unitary supply. Thus, if $D_{k, t}$ is the dividend payed by asset $k \in\{1,2\}$ at the beginning of period $t$ we have

$$
D_{1, t}+D_{2, t}=1, \quad \text { for all }(t, \sigma) \in \mathbb{N} \times \Sigma \text {. }
$$

Assets dividend are perfectly anti-correlated, namely asset 1 (2) pays a dividend only when state one $1(2)$ is realized. We have

$$
D_{k, t}\left(s^{t}\right)=\Delta_{k, s_{t}}, \quad \text { for all }(t, \sigma) \in \mathbb{N} \times \Sigma \text { and } k=1,2,
$$

where $\Delta_{k, j}$ is the Kronecker's delta. State 1 is the good state (good news) for asset 1 and the bad state (bad news) for assets 2 , and the other way around.

In every period each agent uses her wealth, that is, dividends and the resale value of assets bought in the previous period, to consume and invest. We denote with $C_{t}^{i}$ agent $i$ consumption and with $W_{t}^{i}$ agent $i$ pre-consumption wealth, in units of the numérarire, in the generic $(t, \sigma)$. We express both consumption and portfolio decisions in date $t$ as a function of wealth. In particular, $\delta_{t}^{i}$ is the fraction of wealth that agent $i$ saves for investment and $\left(x_{1, t}^{i}, x_{2, t}^{i}\right)$ is his portfolio decision in $(t, \sigma)$. To ensure that consumption is positive in each period and that the budget constraint is satisfied, we assume the following

$\mathbf{R} 1 \delta_{t}^{i} \in(0,1)$ and $x_{1, t}^{i}+x_{2, t}^{i}=1$, for all $(t, \sigma) \in \mathbb{N}_{0} \times \Sigma$ and $i=1,2$.

Naming $P_{t}=\left(P_{1, t}, P_{2, t}\right)$ the vector of date- $t$ asset prices, agent $i$ 's budget constraint in $(t, \sigma)$ is

$$
W_{t}^{i}=\sum_{k=1}^{2}\left(P_{k, t}+\Delta_{k, s_{t}}\right) \frac{\delta_{t-1}^{i} x_{k, t-1}^{i} W_{t-1}^{i}}{P_{k, t-1}}, \quad \text { for } i=1,2 .
$$

Given asset demands, and having assumed unitary supply, prices are determined in equilibrium. Using portfolio decision, the market clearing conditions in $(t, \sigma)$ leads to

$$
P_{k, t}=\delta_{t}^{1} x_{k, t}^{1} W_{t}^{1}+\delta_{t}^{2} x_{k, t}^{2} W_{t}^{2}, \quad \text { for } k=1,2 \text {. }
$$

Equations (4-5) define the coupled dynamics of asset prices and agents' wealth as a function of agents investment and portfolio decisions. Specific assumptions of agents' behavior shall be taken starting from the next section.

Finally, we go back to the dividend process and assume that states of nature follow a Markov process. We take $\mathrm{P}$ as the probability measure on $(\Sigma, \mathcal{F})$ such that the coordinate process $\left(s_{t}\right)_{t=0}^{\infty}$ is Markov with unitary memory and transition matrix

$$
\Pi=\left[\begin{array}{cc}
1 / 2+\lambda & 1 / 2-\lambda \\
1 / 2-\lambda & 1 / 2+\lambda
\end{array}\right], \lambda \in(0,1 / 2)
$$


Given $\Pi$, the invariant distribution is

$$
\pi^{*}=\left[\begin{array}{l}
1 / 2 \\
1 / 2
\end{array}\right]
$$

Having Markov states is the main difference with respect to Evstigneev et al. (2008) and Bottazzi et al. (2017), where states of nature follow a general i.i.d. process. As we shall explain, the difference is key for the emergence of momentum. The i.i.d. case can be recovered by setting $\lambda=0$ in (6).

\subsection{Rational Pricing}

Under homogeneous initial endowment, homogeneous discount factor $\delta .7$ and rational expectations, rational equilibrium prices can be recovered imposing market clearing conditions when portfolio and saving decision of each agent $i=1,2$ solve

$$
\begin{aligned}
& \max _{\delta_{t}^{i}, x_{k, t}^{i} ; \forall k, t, \sigma} \mathrm{E}\left[\sum_{t=0}^{\infty} \delta^{t} U_{i}\left(C_{t}^{i}\right)\right] \quad \text { subject to } \\
& C_{t}^{i}=\left(1-\delta_{t}^{i}\right) W_{t}^{i}, \forall(t, \sigma) \in \mathbb{N}_{0} \times \Sigma ; \\
& W_{t}^{i}=\sum_{k=1}^{2}\left(P_{k, t}+\Delta_{k, s_{t}}\right) \frac{\delta_{t-1}^{i} x_{k, t-1}^{i} W_{t-1}^{i}}{P_{k, t-1}}, \forall(t, \sigma) \in \mathbb{N} \times \Sigma ; \\
& W_{0}^{i}=\frac{1}{2} \sum_{k=1}^{2} P_{k, 0}+\frac{1}{2} ;
\end{aligned}
$$

where we have also assumed that each agent starts with an endowment of $1 / 2$ consumption good. Solving the former for each agent and adding the market clearing (5) leads to equilibrium saving rules, portfolio rules, and asset prices. In particular, a constant aggregate endowment and no-aggregate risk imply that both agents use the same saving rule and hold homogeneous portfolios irrespectively of their per-period utility. Using the superscript RE ("Rational Expectation") to label the quantities involved in this exercise, the saving rate is

$$
\delta_{t}^{\mathrm{RE}}=\delta \quad \text { for all }(t, \sigma) \in \mathbb{N}_{0} \times \Sigma .
$$

and the fraction of wealth invested in asset 1 in $(t, \sigma)$ is

$$
x_{1, t}^{\mathrm{RE}}\left(s_{t}=1\right)=\frac{1 / 2+\lambda-2 \lambda \delta}{1-2 \lambda \delta}, \quad x_{1, t}^{\mathrm{RE}}\left(s_{t}=2\right)=\frac{1 / 2-\lambda}{1-2 \lambda \delta} .
$$

\footnotetext{
${ }^{7}$ The same analysis holds even when agents have a different discount factor. The only difference is that the risk-free rate depends on agents relative wealth. In the long-run, only the agent with the highest discount factor has positive wealth, and thus determines prices.
} 
The amount invested in asset 2 follows by $\mathbf{R} \mathbf{1}$. The price of asset 1 in $(t, \sigma)$ is

$$
P_{1, t}^{\mathrm{RE}}\left(s_{t}=1\right)=\frac{\delta}{1-\delta} \frac{1 / 2+\lambda-2 \lambda \delta}{1-2 \lambda \delta}, \quad P_{1, t}^{\mathrm{RE}}\left(s_{t}=2\right)=\frac{\delta}{1-\delta} \frac{1 / 2-\lambda}{1-2 \lambda \delta} .
$$

Given the symmetry of the model, $P_{2, t}^{\mathrm{RE}}\left(s_{t}=1\right)=P_{1, t}^{\mathrm{RE}}\left(s_{t}=2\right)$ and $P_{2, t}^{\mathrm{RE}}\left(s_{t}=2\right)=$ $P_{1, t}^{\mathrm{RE}}\left(s_{t}=1\right)$. The implied one period risk-free rate is constant and equal to

$$
r_{f}=\frac{1-\delta}{\delta}
$$

If we normalize prices by their sum, the normalized price of asset $k$ jumps between $x_{k}^{\mathrm{RE}}(1)$ and $x_{k}^{\mathrm{RE}}(2)$ depending on the realized state. Having assumed that states follow a Markov process, equilibrium portfolio rules and asset prices are state dependent.

Under rational expectations and with no aggregate risk, fair pricing holds. In fact, for both assets $k=1,2$ we obtain

$$
\mathrm{E}\left[r_{k, t+1} \mid I_{t}\right]=0 \quad \text { for all }(t, \sigma) \in \mathbb{N}_{0} \times \Sigma \quad \text { and for all } I_{t} \in \mathcal{J}_{t}
$$

where $\left(\mathcal{J}_{t}\right)$ is the information filtration generated by prices 8 Equation (11) and the tower property of conditional expectation imply that all the quantities defined in Section 3 concerning momentum and reversal are zero for all dates $t$, orders $j$, and initial prices $P_{t-j+1}$. When all agents are fully rational neither momentum nor reversal can occur in equilibrium.

\subsection{Simple rules and long-run heterogeneity}

Given that momentum and reversal are empirical regularities, we move away from assuming that agents have rational expectations. We do so in three ways.

First, we assume that agents do not use the correct probabilistic model but instead believe that states of the world follow an i.i.d. process. The key feature is that agents underestimate the positive autocorrelation of good and bad news. As we shall show, this is key for generating short-term momentum.

Second, we assume that agents save a constant proportion of their wealth and hold constantly rebalanced portfolios, derived in accordance with their beliefs. This assumptions enables us to give an analytic expression for the stochastic difference equation that generates the equilibrium price process, along the lines of Bottazzi et al. (2017). The expression is crucial for the explicit valuation of conditional expected returns.

\footnotetext{
${ }^{8}$ As we shall characterize later, $\left(\mathcal{J}_{t}\right)$ can be restricted to $\left(\mathcal{F}_{t}\right)$ for our purposes, see the beginning of Section 5 .
} 
Third, we shall assume that beliefs are sufficiently diversified, so that, almost surely, both classes of agents have positive wealth in the long-run. As we shall explain, having persistent heterogeneity is key for generating long-term reversal..$^{9}$

Starting from beliefs, we assume that in every $t \in \mathbb{N}_{0}$, each agent $i=1,2$ assigns a probability $0<\pi^{i}<1$ to the event "asset 1 pays the dividend in date $t+1$ ". $1-\pi^{i}$ is the probability she assigns to the event "asset 2 pays the dividend in $t+1$ ". Moreover, each agent allocates to asset $k$ a fraction of wealth proportional to the likelihood that asset $k$ pays the dividend. Agents have also a constant and homogeneous saving rate equal to $\delta$. Formally

$$
\mathbf{R 2}\left(x_{1, t}^{i}, x_{2, t}^{i} ; \delta_{t}^{i}\right)=\left(\pi^{i}, 1-\pi^{i} ; \delta\right) \text { for all }(t, \sigma) \in \mathbb{N}_{0} \times \Sigma \text { and } i=1,2 .
$$

Without loss of generality, we also assume that $0<\pi^{2}<\pi^{1}<1$. Agent 1 assigns a higher probability than agent 2 to the event that asset 1 pays the dividend, and the other way around for asset 2 .

When the dividend process is i.i.d. and agent beliefs are correct, Evstigneev et al. (2008) name the portfolio rule in $\mathbf{R} 2$ the Generalized Kelly Rule. The rule coincides with the one used in equilibrium by a representative agent with correct beliefs. Bottazzi et al. (2017) analyze such a rule for general beliefs and name it Subjective Generalized Kelly. They show that the resulting wealth and price dynamics is such that prices are strictly positive and exclude arbitrages. Even if this rule is in general not optimal, Bottazzi et al. (2017) link it with an endogenous intertemporally optimal rule using the concept of effective beliefs. Basically, the price process implied by agents behaving in accordance to this rule is the same as in a log-economy where agents' beliefs are affected by asset prices, the higher the price the higher the belief that the asset pays the dividend.

Since agents' saving rate $\delta$ matches the agents' discount factor in the rational expectation case, the equilibrium risk-free rate is still (10). Moreover, the total wealth in the model sums up to $1 /(1-\delta)$ and prices sum up to $\delta /(1-\delta)$. Defining $p_{t}=P_{1, t}(1-\delta) / \delta$ as the normalized price of asset 1 and $w_{t}=W_{t}^{1}(1-\delta)$ as the normalized wealth of agent 1, under $\mathbf{R} 2$ the dynamics of wealth and prices in (45) can be rewritten as

$$
\left\{\begin{array}{l}
w_{t}=w_{t-1}\left(\frac{\delta p_{t}+(1-\delta) \Delta_{1, s_{t}}}{p_{t-1}} \pi^{1}+\frac{1-\delta p_{t}-(1-\delta) \Delta_{1, s_{t}}}{1-p_{t-1}}\left(1-\pi^{1}\right)\right) \\
p_{t}=\pi^{1} w_{t}+\pi^{2}\left(1-w_{t}\right)
\end{array}\right.
$$

Using both equations, we find the analytic expression of the stochastic difference

\footnotetext{
${ }^{9}$ The same intuition does generally apply to all models characterized by long-run heterogeneity, see the discussion below Corollary 4.1 .
} 


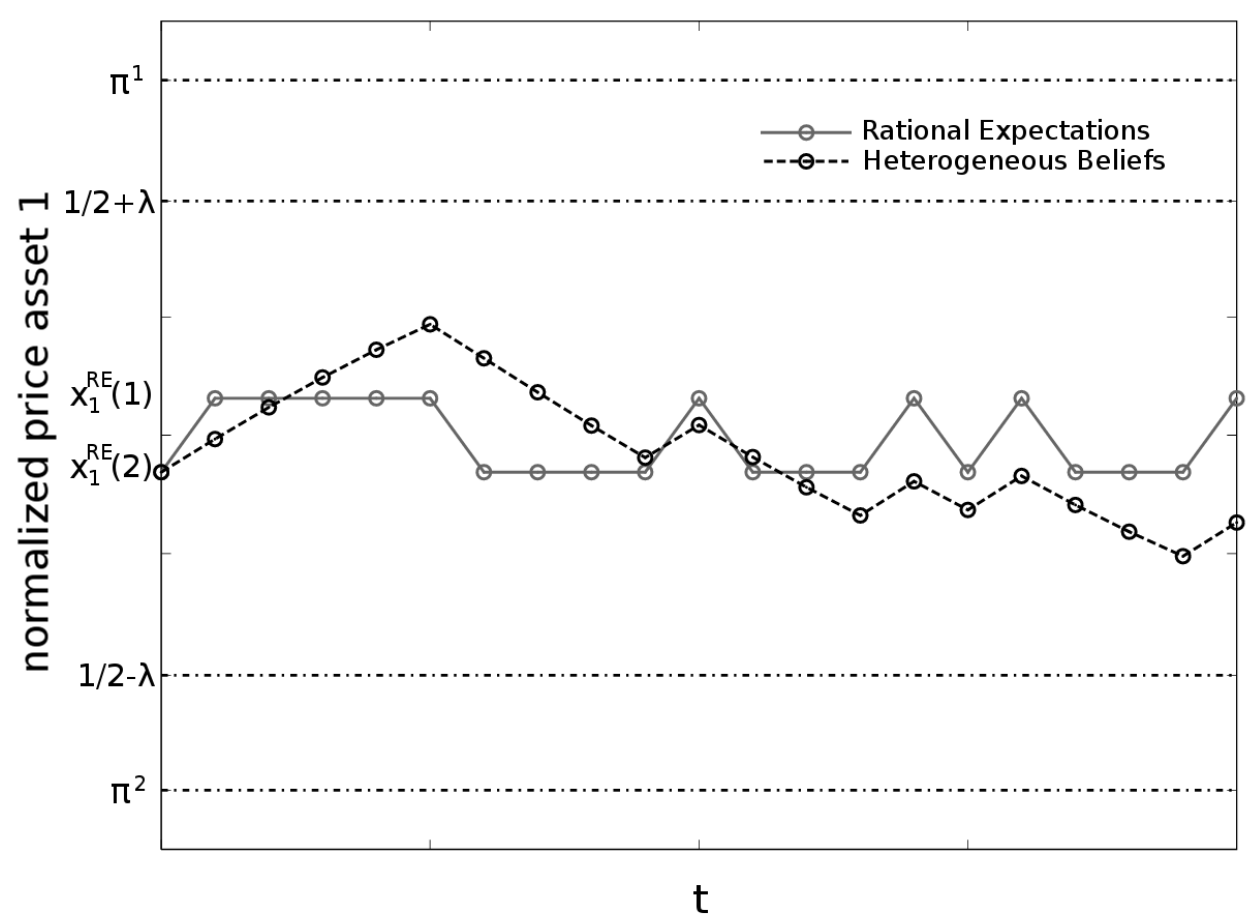

Figure 1: Comparison of price patterns for a given realization of the underlying process. The values used in the plot are $\pi^{1}=0.8, \pi^{2}=0.2, \delta=0.9$ and $\lambda=0.2$.

equation that generates the price process $\left(p_{t}\right)_{t \in \mathbb{N}_{0}}$ under belief heterogeneity

$$
p_{t}=p_{t-1}+\frac{(1-\delta)\left(\pi^{1}-p_{t-1}\right)\left(p_{t-1}-\pi^{2}\right)\left(\Delta_{1, s_{t}}-p_{t-1}\right)}{p_{t-1}\left(1-p_{t-1}\right)-\delta\left(\pi^{1}-p_{t-1}\right)\left(p_{t-1}-\pi^{2}\right)}
$$

Figure 1 shows the (normalized) price patterns of asset 1 for a representative path $\sigma$ both when agents have rational expectations, eq. (9), and when agents invest according to R2, eq. (12). Prices generated by (12) fluctuate in the interval $\left(\pi^{2}, \pi^{1}\right)$ depending on the realized dividend. When $s_{t}=1$, eq. (12) implies that the price of asset 1 increases while the price of asset 2 decreases. Since we have assumed that $\pi^{1}>\pi^{2}$, agent 1 has a higher stake in asset 1 than agent 2 . Therefore, a good news for asset 1 increases agent 1's relative wealth and thus pushes asset 1 price up. The contrary happens when $s_{t}=2$. Starting from the same price $x_{1}^{\mathrm{RE}}(2)$, consider an interval of 5 good news for asset 1 . If all agents are rational, the (normalized) price jumps in the first period to $x_{1}^{\mathrm{RE}}(1)$ and remains there until bad states arrive. If, instead, agents follow $\mathbf{R} 2$, the price keeps increasing because agent 1 keeps profiting from the trade. For the first two periods the price stays below the rational one, an effect that could be referred to as market under-reaction. For the subsequent 3 periods, however, the market seems to over-react. If 4 bad states 
for asset 1 follow, agent 2 profits from the trade and the price slowly decreases, showing a form of market under-reaction again.

According to the stochastic difference equation (12), the size of price changes depends on the saving rule $\delta$ and on beliefs $\pi^{1}$ and $\pi^{2}$. Without a further restriction on beliefs and saving rates, it could happen that, on average, the price increases (decreases) so that eventually $p_{t}$ approaches $\pi^{1}\left(\pi^{2}\right)$, meaning that only agent 1 (2) survives in the long run. Alternatively, beliefs could be such that both agents survive in the long run and prices never settles down. As we shall see, in order to have reversal it is key that both agents survive. The following proposition gives conditions on beliefs and saving rates such that both agents have strictly positive wealth in the long-run, P-a.s., and prices never settle down.

Proposition 4.1. Under R1-R2, if agents saving rates $\delta$ and beliefs $\pi^{1}$ and $\pi^{2}$ are such that, for every $i, j \in\{1,2\}$ with $i \neq j$,

$$
\left(\frac{1}{2}+\lambda\right) \log \left(\frac{\delta \pi^{j}+(1-\delta) \pi^{i}}{\pi^{j}}\right)+\left(\frac{1}{2}-\lambda\right) \log \left(\frac{1-\delta \pi^{j}-(1-\delta) \pi^{i}}{1-\pi^{j}}\right)>0
$$

and

$$
\left(\frac{1}{2}-\lambda\right) \log \left(\frac{\delta \pi^{j}+(1-\delta) \pi^{i}}{\pi^{j}}\right)+\left(\frac{1}{2}+\lambda\right) \log \left(\frac{1-\delta \pi^{j}-(1-\delta) \pi^{i}}{1-\pi^{j}}\right)>0,
$$

then both agents survive:

$$
\operatorname{Prob}\left\{\limsup _{t \rightarrow \infty} w_{t}>0 \text { and } \liminf _{t \rightarrow \infty} w_{t}<1\right\}=1 .
$$

Moreover,

$$
\operatorname{Prob}\left\{\liminf _{t \rightarrow \infty} p_{t}=\pi^{2} \text { and } \limsup _{t \rightarrow \infty} p_{t}=\pi^{1}\right\}=1 .
$$

Proof. See Appendix A.

Thus, in order to obtain long-run heterogeneity, we shall assume the following.

R3 Given $\lambda$, beliefs $\pi^{1}, \pi^{2}$, and the saving rate $\delta$ are such that the inequalities in (13) and (14) are satisfied.

Intuitively $\mathbf{R} 3$ is satisfied when agent 1 overestimates the probability that asset 1 pays the dividend while agent 2 overestimates the probability that asset 2 pays the dividend. Stated in terms of portfolios, if both agents survive then their positions must be sufficiently diversified. 
Corollary 4.1. Condition R3 implies that, for any given $\delta \in(0,1)$ and $\lambda \in$ $(0,1 / 2)$, it is $\pi^{1}>1 / 2+\lambda$ and $\pi^{2}<1 / 2-\lambda$.

Proof. See Appendix B.

As surveyed in Section 2, the incumbent market selection literature has investigated other conditions under which heterogeneity is persistent in financial markets, such as incomplete markets, solvency constraints, non-tradable income, non-converging learning, ambiguity aversion, or recursive preferences. While technically different, all these conditions lead to similar effects on assets returns. The advantage of deriving persistent heterogeneity assuming constantly rebalanced portfolios, $\mathbf{R 2}$, is that the dynamics of prices, eq. (12), can be explicitly derived. Such expression simplifies the computation of conditional expected returns and allows for analytic results.

\section{Momentum and Reversal}

In this section, we use the characterization of the normalized price process $\left(p_{t}\right)$ in (12) to show that prices exhibit short-run momentum if dividends are positively correlated, $\lambda>0$, and exhibit long-run reversal if agents' heterogeneity is persistent. Given the symmetry of our model we shall focus on asset 1. An important preliminary observations is the following

Lemma 5.1. If $s_{t}=1$ then $r_{1, t}>0$ and $r_{2, t}<0$ while if $s_{t}=2$ then $r_{1, t}<0$ and $r_{2, t}>0$

Proof. Since the sum of the prices of the two assets is constant and equal to the inverse of the interest rate, the excess return of the two assets must have opposite sign. Let consider asset 1 . In terms of rescaled variables

$$
r_{1, t}=\frac{p_{t}-p_{t-1}}{p_{t-1}}+\frac{1-\delta}{\delta}\left(\frac{\Delta_{1, s_{t}}}{p_{t-1}}-1\right) .
$$

According to 12 , the difference $p_{t}-p_{t-1}$ is positive when $s_{t}=1$ and negative when $s_{t}=2$. The same is true for $\Delta_{1, s_{t}} / p_{t-1}-1$ and the statement follows.

Since a positive (negative) excess return for asset 1 in equivalent to observing the realization of state 1 (state 2 ), both sets $\mathcal{R}_{t}^{+}(j)$ and $\mathcal{R}_{t}^{+}(j)$ are elements of the primitive information filtration $\mathcal{F}_{t}$ other than of $\mathcal{J}_{t}$. In other terms, conditioning on the realization of a sequence of positive (negative) returns of asset 1 is equivalent to conditioning on the realization of a sequence of states of the world 1 (2). The opposite for asset 2 .

We begin with the analysis of the simple case of agents having the same beliefs. The same analysis is also informative of the case in which beliefs are such that, eventually, only one agent has positive wealth. 


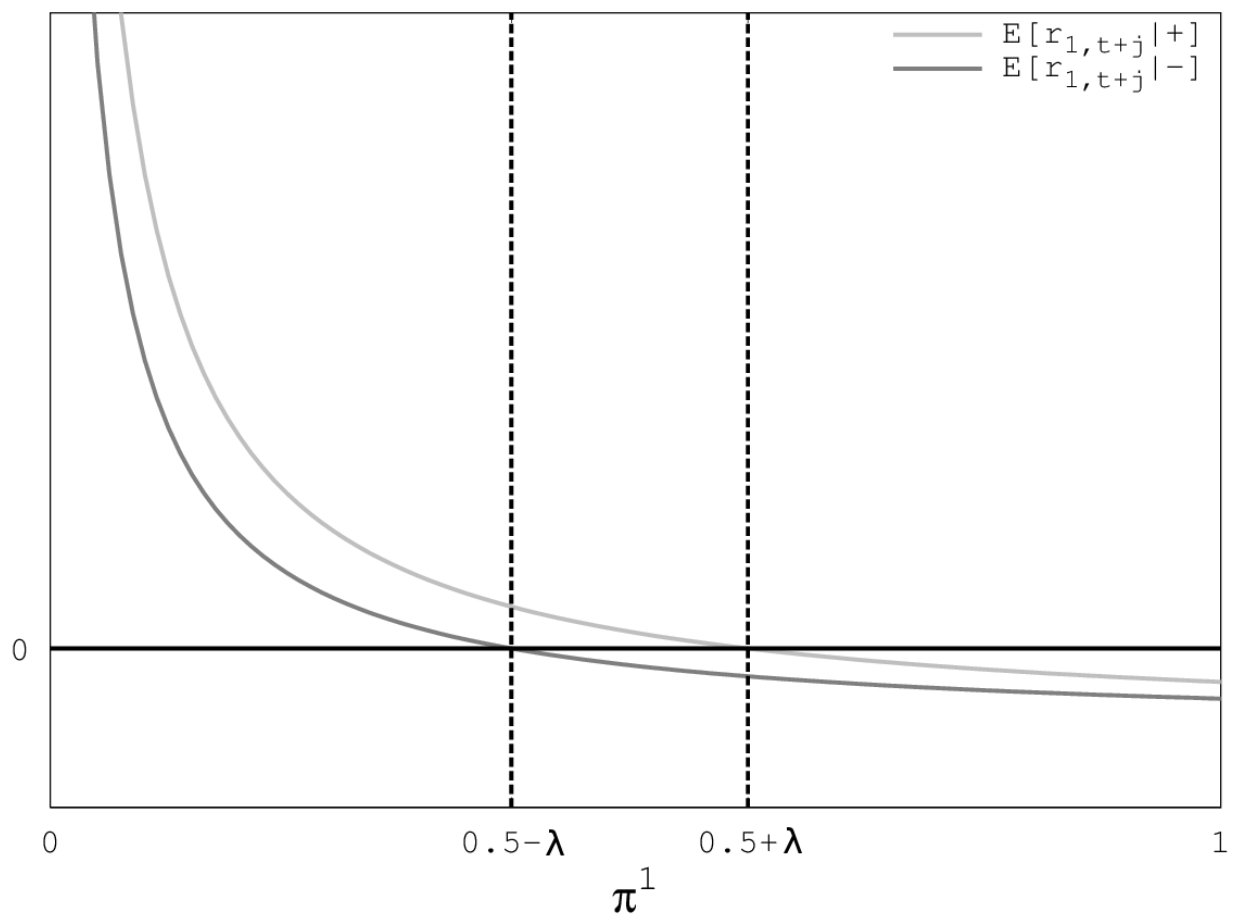

Figure 2: Comparison of asset 1's expected excess returns for different values of $\pi^{1}$ in the case of Homogeneous beliefs. The values used in the plot are $\delta=0.95$ and $\lambda=0.1$.

\subsection{Homogeneous beliefs}

Set $\pi^{2}=\pi^{1}$ in $(12)$ to obtain $p_{t}=\pi^{1}$ for all $(t, \sigma)$. In this case only the last return matters, as it determines the probability of good or bad news. Thus there is no difference in conditioning on longer histories of returns with the same sign. Hence for any $j$, the expected return of asset 1 reads

$$
\begin{aligned}
& \mathrm{E}\left[r_{1, t+1} \mid \mathcal{R}_{t}^{+}(j), \pi^{1}\right]=\frac{1-\delta}{\delta}\left(\frac{1 / 2+\lambda}{\pi^{1}}-1\right), \\
& \mathrm{E}\left[r_{1, t+1} \mid \mathcal{R}_{t}^{-}(j), \pi^{1}\right]=\frac{1-\delta}{\delta}\left(\frac{1 / 2-\lambda}{\pi^{1}}-1\right) .
\end{aligned}
$$

Figure 2 shows a representative example of the two quantities as functions of $\pi^{1}$. In this economy there is no capital gain (prices are constant), hence the expected excess return is just the expected dividend yield, a decreasing function of the price, minus the risk-free rate. Given assets' dividend process, the expected dividend yield is proportional to conditional probabilities. It follows that the excess return 
after a given state hits zero when $\pi^{1}$ matches the probability that asset 1 pays the dividend after that state. Thus, when $1 / 2-\lambda<\pi^{1}<1 / 2+\lambda$ one has momentum both after positive returns and after negative returns. When, instead, $\pi^{1}<1 / 2-\lambda$ one has momentum after positive returns and reversal after negative returns. The opposite occurs when $\pi^{1}>1 / 2+\lambda$.

Despite for extreme beliefs both momentum and reversal are possible, depending on the sign of the past return, the size of each expected return is such that their averages $\phi_{t}\left(j, \pi^{1}\right)$ and $\Phi_{t}(j)$ are positive. The result is momentum of all order $j$ and for any belief $\pi^{1}$.

Proposition 5.1. If agents behave according to $\mathbf{R} \mathbf{1}-\mathbf{R} \mathbf{2}$ with $\pi^{1}=\pi^{2}$, then

$$
\phi_{t}\left(j, \pi^{1}\right)=\frac{1-\delta}{\delta} \frac{\lambda}{\pi^{1}}>0 \quad \text { for all } t \in \mathbb{N}, j \leq t, \pi^{1} \in(0,1)
$$

and

$$
\Phi_{t}(j)=\phi_{t}\left(j, \pi^{1}\right)>0 \quad \text { for all } t \in \mathbb{N}, j \leq t, \pi^{1} \in(0,1) .
$$

Proof. Due to the nature of the dividend process, the probability to observe a sequence of $j$ identical states is identical for the two states. For Lemma 5.1 this also applies to a sequence of concordant returns, so that

$$
\phi_{t}\left(j, \pi^{1}\right)=\frac{1}{2} \mathrm{E}\left[r_{1, t+1} \mid \mathcal{R}_{t}^{+}(j), \pi^{1}\right]-\frac{1}{2} \mathrm{E}\left[r_{1, t+1} \mid \mathcal{R}_{t}^{-}(j), \pi^{1}\right] .
$$

The price distribution in this case is atomic: using the indicator function it is $F_{t}(p)=I_{p>\pi^{1}}$. Thus $\Phi_{t}(j)=\phi_{t}\left(j, \pi^{1}\right)$.

\subsection{Heterogeneous Beliefs}

Conditional expected returns in homogeneous economies are not consistent with the empirical evidence. We would like to have both momentum and reversal, but for different values of $j$. Thus, in what follows, we restrict the investigation to beliefs satisfying $\mathbf{R} 3$ so that heterogeneity is persistent and prices keep fluctuating as shown in Figure 1. To retain analytic tractability we shall consider two specific cases: $j=1$, as a proxy of short-run, and $j \rightarrow \infty$, as a proxy of long-run.

Short-run. The expressions for $\mathrm{E}\left[r_{1, t+1} \mid \mathcal{R}_{t}^{+}(1), p_{t}\right]$ and $\mathrm{E}\left[r_{1, t+1} \mid \mathcal{R}_{t}^{-}(1), p_{t}\right]$ can be directly obtained from (12). Figure 3 reports their typical dependence on the price $p_{t}$. Notice that $\mathrm{E}\left[r_{1, t+1} \mid \mathcal{R}_{t}^{+}(1), p_{t}\right]$ is positive if $p_{t}<1 / 2+\lambda$ and negative otherwise, meaning that one has momentum after a positive return when $p_{t}$ smaller than $1 / 2+\lambda$ and reversal otherwise. The situation is symmetric in the case of a 


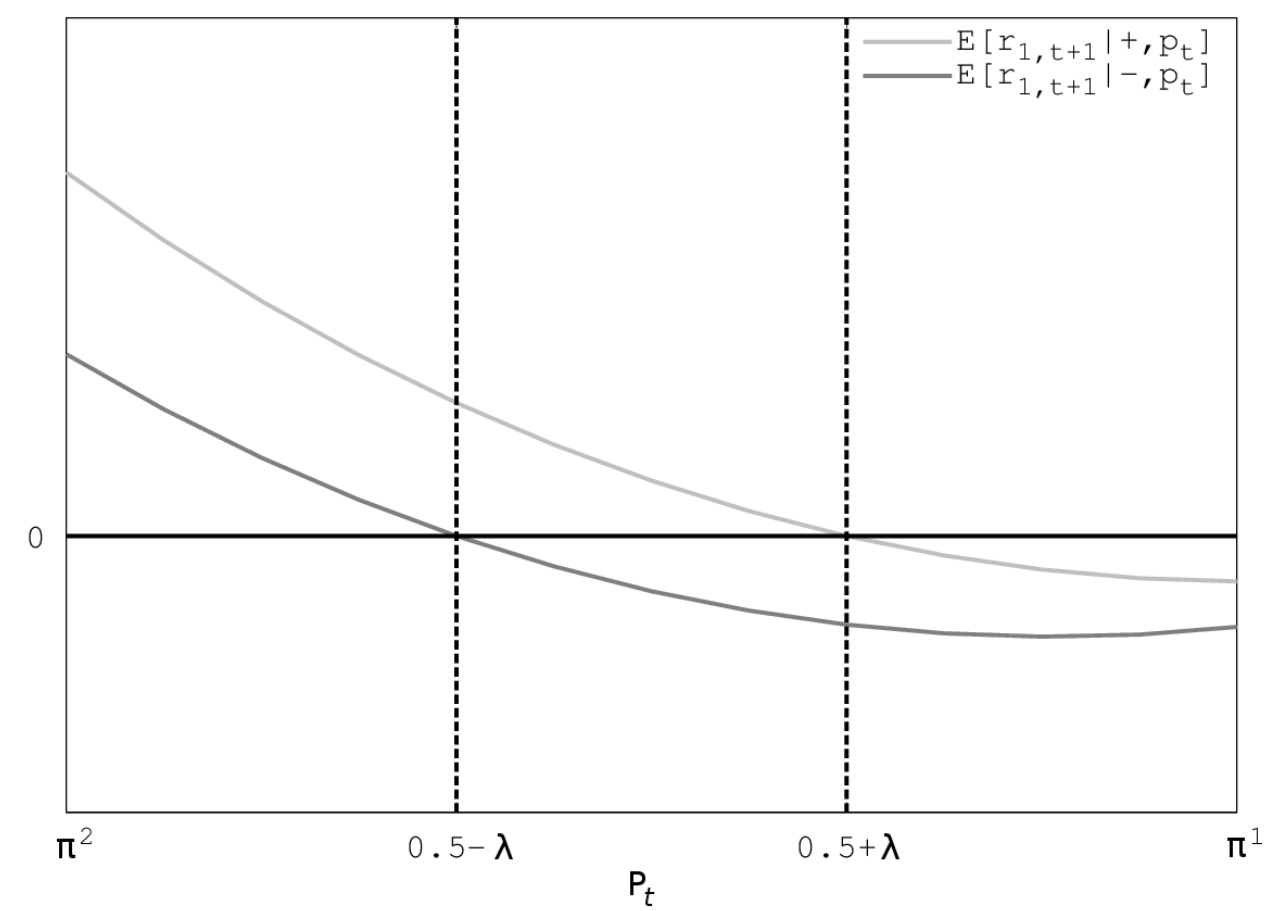

Figure 3: $\mathrm{E}\left[r_{1, t+1} \mid \mathcal{R}_{t}^{+}(1), p_{t}\right]$ and $\mathrm{E}\left[r_{1, t+1} \mid \mathcal{R}_{t}^{-}(1), p_{t}\right]$ for different values of $p_{t}$. The values used in the plot are $\pi^{1}=0.8, \pi^{2}=0.2, \delta=0.95$ and $\lambda=0.1$.

negative return: momentum occurs if $p_{t}>1 / 2-\lambda$ and reversal if $p_{t}<1 / 2-\lambda$. Thus for values of $p_{t} \in(1 / 2-\lambda, 1 / 2+\lambda)$ one has momentum of order 1 both after a positive return and after a negative return, while for $p_{t}$ outside such interval one has momentum of order 1 only for positive or negative past returns, depending on the exact position of $p_{t}$, and reversal otherwise. However, even when $p_{t}<1 / 2-\lambda$ or $p_{t}>1 / 2+\lambda$, the size of momentum is larger than the size of reversal. Thus, when we average over past histories of returns to get $\phi_{t}\left(1, p_{t}\right)$ and $\Phi_{t}(1)$, the momentum effect prevails.

Proposition 5.2. If agents behave according to $\mathbf{R} \mathbf{1}-\mathbf{R} 3$, then

$\phi_{t}\left(1, p_{t}\right)=\frac{1-\delta}{\delta} \frac{\lambda\left(1-p_{t}\right)}{p_{t}\left(1-p_{t}\right)-\delta\left(\pi^{1}-p_{t}\right)\left(p_{t}-\pi^{2}\right)}>0 \quad$ for all $t \in \mathbb{N}, p_{t} \in\left(\pi^{1}, \pi^{2}\right)$, and

$$
\Phi_{t}(1)>0 \quad \text { for all } t \in \mathbb{N}
$$

Proof. See Appendix C. 
We have established that a financial market populated by heterogeneous agents exhibits short-run momentum. Under agents' heterogeneity two are the forces driving this result. The dividend yield, as in the homogeneous agent model of Figure 2, and the capital gain. Provided prices are close to rational values and in the bound $(1 / 2-\lambda, 1 / 2+\lambda)$, both forces push in the same direction: A dividend payment is more likely to be followed by a dividend payment; and a price increase is more likely to be followed by a price increase (and the other way round). Moreover, the dividend payment effect is stronger that the price increase effect, so that a high (low) dividend yield is more likely to be followed by high (low) dividend yield.

For positive returns, the momentum effect of dividend yield breaks when prices are too far form their rational values, beyond $1 / 2+\lambda$. When prices are too large, the dividend yield becomes too low and prices do not grow that much anymore. However, in this case, the effect of negative news on momentum is quite strong, as a negative return is more likely to be followed by a quite large negative return, so that the momentum effect of the capital gain component becomes stronger. The combined result is still momentum. The same argument holds for negative returns when prices are too low. In this case, it is the contribution of positive returns that leads to momentum.

Short-run momentum is decreasing in $\delta$. An increase in agents saving rate decreases the interest rates, thus increasing prices and decreasing dividend yields. Moreover, an increase in agents saving rate decreases the size of price jumps: agents effective portfolio positions become more and more alike and speculative trade diminishes 10

Long-run. We turn to expected returns in the long-run. In the limit of the length of histories being $+\infty$, the initial normalized price $p_{t}$ does not play any role. Indeed for Lemma 5.1, as the length of the sequence of good (bad) news increases, the price tends toward its upper bound $\pi^{1}$ (lower bound $\pi^{2}$ ). Thus, the expected excess returns after a sufficiently long sequence of good or bad news are $\mathrm{E}\left[r_{1, t+1} \mid \mathcal{R}_{t}^{+}(1), \pi^{1}\right]$ and $\mathrm{E}\left[r_{1, t+1} \mid \mathcal{R}_{t}^{-}(1), \pi^{2}\right]$, respectively. Consider again Fig. 3 . Provided beliefs obey to R3, Corollary 4.1 guarantees that $\pi^{1}>1 / 2+\lambda$ and $\pi^{2}<1 / 2-\lambda$. Thus the price process exhibits reversal both after a very long sequence of positive news and after a very long sequence of negative news. The intuition is that after a long-sequence of positive news prices are close to $\pi^{1}$ and

\footnotetext{
${ }^{10}$ For a precise appraisal of the role of saving rates on agents' portfolio positions we refer the reader to the discussion on effective beliefs in Bottazzi et al. (2017). Intuitively, simple portfolio rules can be matched into log-optimal portfolio rules by modifying agents' beliefs into effective beliefs. Higher values of saving rates imply a low interest rate and thus a high influence of assets' future dividends for their evaluation. The same pair of simple rules need closer effective beliefs, and thus less severe speculation, in order to counterbalance the higher impact of future dividends.
} 

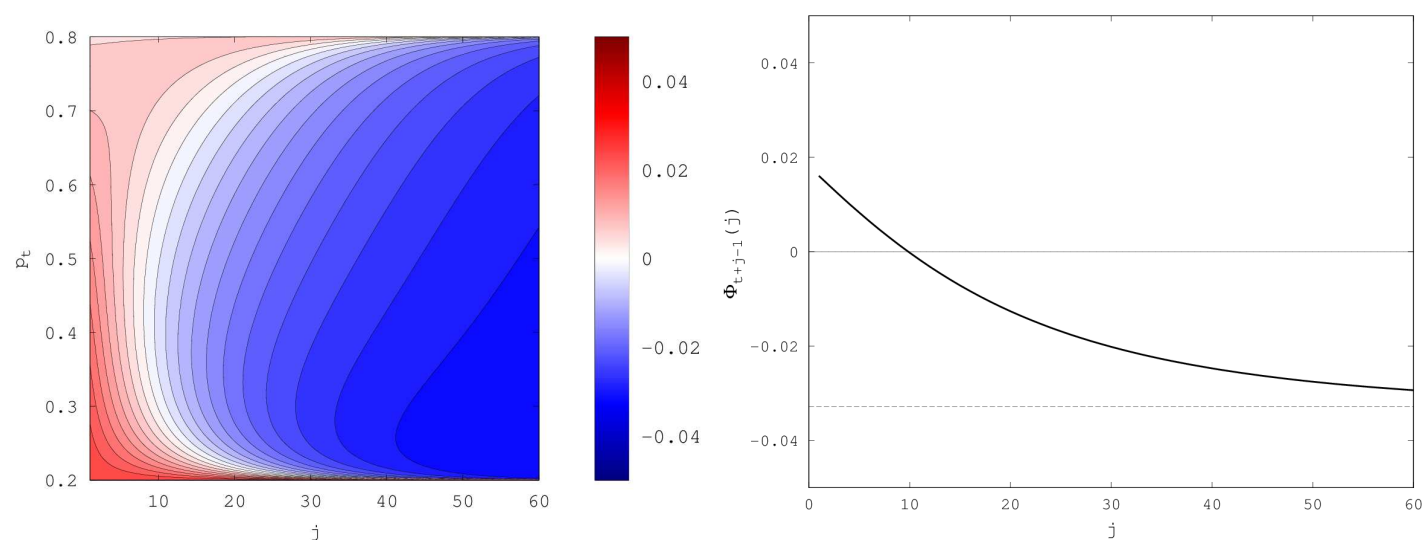

Figure 4: Left panel: $\phi_{t+j-1}\left(j, p_{t}\right)$ for different values of $j$ and $p_{t}$. Right panel: $\Phi_{t+j-1}(j)$ for $j=1,2, \ldots, 60$ with $t=10000, N=1000000, \delta=0.95, \pi^{1}=$ $0.8, \pi^{2}=0.2$ and $\lambda=0.1$. The dashed line represents the theoretical value for $\Phi_{\infty}$.

the asset is overpriced, thus making the expected dividend yield low. Moreover, given that we have imposed conditions for long-run heterogeneity, $\mathbf{R 3}$, the price is a mean reverting process: when price are close to $\pi^{1}$ negative jumps are stronger than positive jumps and expected capital gains are negative. The two effect together lead to reversal, $\mathrm{E}\left[r_{1, t+1} \mid \mathcal{R}_{t}^{+}(1), \pi^{1}\right]<0$. A similar effect occurs after a sequence of negative news. In this case prices are close to $\pi^{2}$ so that the asset is underpriced. Both dividend yields and capital gains are quite large in expectation and the result is still reversal, $\mathrm{E}\left[r_{1, t+1} \mid \mathcal{R}_{t}^{-}(1), \pi^{2}\right]>0$.

Proposition 5.3. If agents behave according to $\mathbf{R} \mathbf{1}-\mathbf{R} 3$, then

$$
\Phi_{\infty}=\phi_{\infty}\left(p_{t}\right)=\frac{1-\delta}{2 \delta}\left(\frac{1 / 2+\lambda}{\pi^{1}}-\frac{1 / 2-\lambda}{\pi^{2}}\right)<0, \text { for all } t \in \mathbb{N}
$$

Proof. See Appendix D.

Having long-run heterogeneity is crucial for the result because it links the occurrence of long histories of returns with the same sign to waves of severe mispricing. In our set-up, waves of mispricing are caused by the wealth accumulation process when agents have incorrect, and diverse enough, beliefs. A sequence of positive news gives more weight to agents with optimistic beliefs while a sequence of negative news gives more weights to agents with pessimistic beliefs.

Numerical exploration. The price evolution (12) can be iterated to compute the functions $\phi_{t+j-1}\left(j, p_{t}\right)$ for any $j$. In Figure 4 , we report the result for $\delta=0.95$, $\pi^{1}=0.8, \pi^{2}=0.2$ and $\lambda=0.1$. Momentum occurs in the short-run for any $p_{t}$. 
As the order $j$ increases, the size of momentum decreases, until reversal appears. The magnitude of the effects and the order $j$ after which reversal appears depend on the initial price $p_{t}$. Since the values used for the example in the left panel of Figure 4 satisfy R3, prices fluctuate between $\pi^{2}$ and $\pi^{1}$. Thus we can compute $\Phi_{t+j-1}(j)$ using numerical techniques to obtain the cumulative distribution of $p_{t}$. We iterate the map in (12) for $t$ steps with initial condition $1 / 2$, record the last value $p_{t}$, and repeat the procedure for $N$ independent runs. As displayed in the right panel of Figure 4, our economy exhibits momentum in the short-run, namely $\Phi_{t+j-1}(j)$ is positive for low values of $j$. As $j$ increases $\Phi_{t+j-1}(j)$ decreases. The expected return becomes negative around $j=10$, implying long-run reversal.

The intuition that underlies our analysis is that short-term momentum and long-term reversal are due to the interplay between two forces: the dividend positive autocorrelation that generates momentum (continuation effect) and the extreme and persistent mispricing that generates reversal (reversal effect). While the continuation effect is constant with respect to $j$, the reversal effect increases with $j$, since for a larger $j$ the mispricing becomes higher. Hence for low values of $j$ the continuation effect overwhelms the reversal effect and the result is short-term momentum. Conversely, for large values of $j$, the reversal effect prevails and the outcome is long-term reversal.

The presence of both momentum and reversal can be explained considering agents relative wealth dynamics, the dividend process, and agents' diverse beliefs. First of all notice that when a good state arrives, the agent who is investing the most in that asset increases her wealth, generating a positive price variation and, provided the asset is not heavily mispriced, a positive excess return. In the case of bad states, the opposite occurs. The positive autocorrelation after good news implies that probability of observing a variation of agents' wealth with a given sign after a variation of the same sign is higher than a variation of opposite sign. This implies that the excess expected return has the same sign of the previous excess return, implying momentum in the short-run. Contrarily, when we consider long series of states of the same type, we have, both in the case of good or bad states, situations of extreme mispricing because one of the two agents has almost all the wealth and prices converge to her beliefs. Here, provided beliefs are diverse enough to guarantee long-run heterogeneity $(\mathbf{R} 2-\mathbf{R} 3)$, it is the poorest agent who is favored. By investing a large enough fraction of her wealth in the underpriced asset and a small enough fraction in the overpriced asset, she is able to grow more in expectation, as ensured by (13) and (14). The result is an expected excess return with opposite sign with respect to the previous ones and long-run reversal to the mean. 


\section{Implications}

Here, we discuss the implications of our model along different dimensions. First, in Section 6.1, we replicate some of the empirical tests of short-term momentum and long-term reversal discussed in the literature using the synthetic data produced by our model. In Section 6.2, we show how there exists a profitable portfolio that, without resorting to short-selling, can provide a positive expected excess return in every period. In Section 6.3, we demonstrate that, when short-selling is allowed, it is possible to build a zero-cost portfolio strategy with positive expected payoffs in every period. Finally in Section 6.4 we explore the sensitivity of momentum and reversal effects to changes in beliefs dispersion and dividend autocorrelation.

\subsection{Replication of Empirical Results}

Having two assets in the economy allows to investigate whether our model is able to replicate the performances of the Winners and Losers portfolios found by empirical literature. In particular, we consider the methodology of De Bondt and Thaler (1985) and apply it to the synthetic data produced by the model.

We start by establishing a length $J$ for the formation period, a length $H$ for the performance period, and the total number of formation periods $N$. Then we numerically iterate the map in $(12)$ for $T=J \times N+H$ periods starting with initial condition ${ }^{11} p_{0}=1 / 2$ and compute for any time step $t=1,2, \ldots, T$ the abnormal return of each asset $k$ defined as

$$
A R_{k, t}=r_{k, t}-\frac{r_{1, t}+r_{2, t}}{2}
$$

For each formation period $n=1, \ldots, N$, we compute the cumulative return of each asset $k$ in that formation period as

$$
C U_{k, n}=\sum_{\tau=(n-1) J+1}^{n J} A R_{k, \tau}
$$

During the same time steps we rank the two assets on the basis of their cumulative return and assign them either to the Winners portfolio (W) or to the Losers portfolio (L). The Winners portfolio built after the $n$-th formation period buys asset 1 if $C U_{1, n}>C U_{2, n}$ and asset 2 if $C U_{2, n}>C U_{1, n}$. The Losers portfolio does the opposite. Then we evaluate the performance of the two portfolios during the following $H$ periods computing the cumulative abnormal return for each $h=1,2, \ldots, H$. Using the subscript $w$ to indicate the winner asset and the subscript $l$ to indicate

\footnotetext{
${ }^{11}$ Different initial conditions do not affect the results.
} 
the loser one, the cumulative abnormal returns for each time step $h$ of the $n$-th performance period read

$$
C A R_{n, h}^{W}=\sum_{\tau=n J+1}^{n J+h} A R_{w, \tau}, \quad C A R_{n, h}^{L}=\sum_{\tau=n J+1}^{n J+h} A R_{l, \tau} .
$$

De Bondt and Thaler (1985), relying on a wide cross-section of assets, prescribes to build Winners and Losers portfolio considering only those assets that have extreme cumulative returns with respect to such cross-section. Having only two assets in our economy, we reproduce such feature using the time series dimension instead of the cross-section one. Knowing the time distribution of cumulative returns, we consider the performance of the two portfolios only after formation periods in which the cumulative return of one asset is below the first quartile (and thus it contributes to the loser portfolio) or above the third quartile (and thus it contributes to the winner portfolio). Naming $M$ the set of formation periods for which the condition is respected, whose cardinality is $N / 2$ given the use of quartiles, we compute the average cumulative abnormal return of each portfolio

$$
A C A R_{h}^{W}=\frac{\sum_{n \in M} C A R_{n, h}^{W}}{N / 2}, \quad A C A R_{h}^{L}=\frac{\sum_{n \in M} C A R_{n, h}^{L}}{N / 2},
$$

and the relative standard errors

$$
S E_{h}^{W}=\sqrt{\frac{\sum_{n \in M}\left(C A R_{n, h}^{W}-A C A R_{h}^{W}\right)^{2}}{N / 2(N / 2-1)}}, \quad S E_{h}^{L}=\sqrt{\frac{\sum_{n \in M}\left(C A R_{n, h}^{L}-A C A R_{h}^{L}\right)^{2}}{N / 2(N / 2-1)}} .
$$

Since in our model dividend are paid in each time step, we assume that one period of our economy corresponds to a quarter. Thus, with $J$ between 12 and 20 we replicate the exercise of De Bondt and Thaler (1985, 1987), while with $J$ between 1 and 4 we replicate the exercise of Jegadeesh and Titman (1993, 2001). $H$ is kept fixed to 20 such that we can observe both short-run and longrun performances. We also calibrate the parameters of the model in such a way that our risk free rate is close to the average 3-month risk free rate recorded in real data and the average dividend yield of our model matches the average quarterly dividend yield recorded empirically ${ }^{12}$ Following such procedure we set $\delta=0.99, \lambda=0.1, \pi^{1}=0.95, \pi^{2}=0.05$. Finally, we choose $N=100000$. Figure

\footnotetext{
${ }^{12}$ Sources of the data: http://www.multpl.com/s-p-500-dividend-yield/ for the dividend yield (whose yearly average is divided by 4 to get the quarterly one) and http://mba. tuck.dartmouth.edu/pages/faculty/ken.french/data_library.html for the 1-month risk free rate (which is compounded in order to find the 3 -month one).
} 

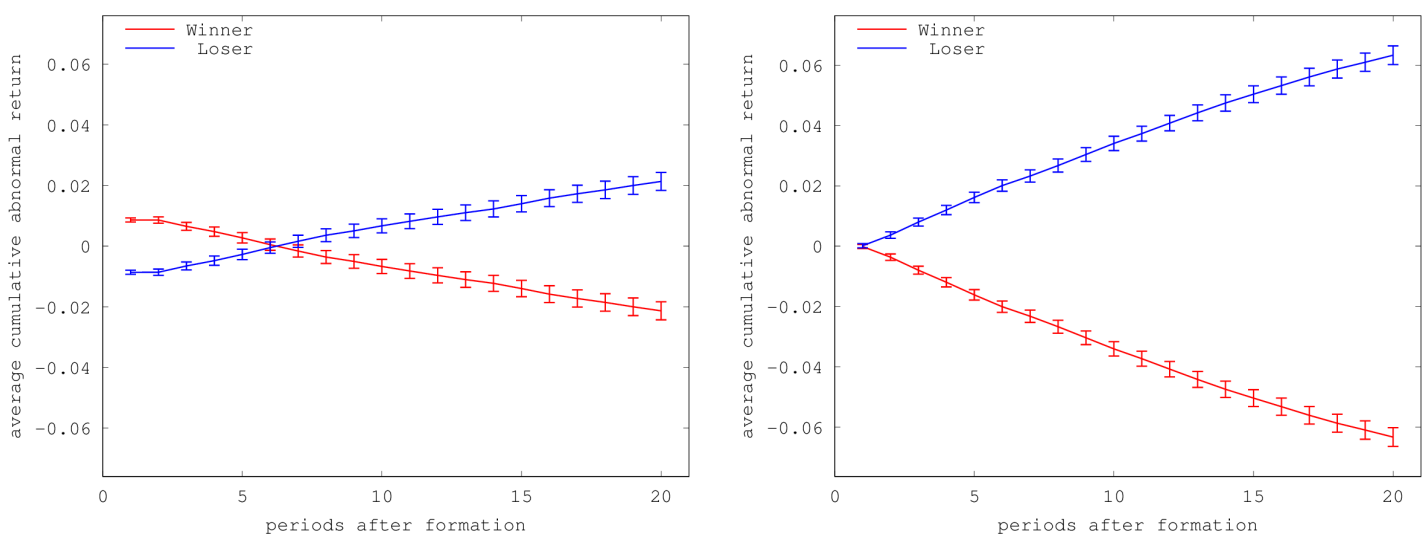

Figure 5: Left panel: $J=2$. Right panel: $J=12$. Error bars are set to three standard errors away from the estimates.

5 shows the results of the exercise for $J=2$ and $J=12$. As one can notice from the left panel, when the portfolios are built looking only at the last 2 periods, in the 5 periods following the formation date the Winners portfolio outperforms the Losers portfolio in a statistically significant way, matching quite well the empirical results of Jegadeesh and Titman 1993, 2001). Moreover, in the subsequent periods the Loser portfolio overcomes the Winners portfolio, reproducing the result of Jegadeesh and Titman (2001) about the long-run reversion of momentum profits. In the right panel, instead, one notices that building the two portfolios on the basis of a formation period of length 12 the Losers portfolio is able to outperform the Winners one in a statistically significant way after 1 period from the formation date. This matches the results of De Bondt and Thaler (1985, 1987) about long-run reversion.

\subsection{Momentum-Reversal Strategy with Short-Selling Con- straint}

When the price process is determined by a representative agent with rational expectations, as we consider in Section 4.1, fair pricing holds and all assets have the same expected return equal to the risk free rate. Thus, any portfolio built using those assets has also zero expected excess return. Provided investors have heterogeneous beliefs and heterogeneity is persistent, the situation is different. Indeed, we have just seen that Winner or Losers potfolios lead to significant profits/losses. In this and the next subsection we show how to improve upon these portfolios.

We start with porfolios that do not allow for short selling. Using the information provided by Figure 3 and considering the symmetry of asset 2 s expected 


\begin{tabular}{c|c|c|c} 
& $p_{t}<1 / 2-\lambda$ & $1 / 2-\lambda<p_{t}<1 / 2+\lambda$ & $p_{t}>1 / 2+\lambda$ \\
\hline$s_{t}=1$ & buy asset 1 & buy asset 1 & buy asset 2 \\
\hline$s_{t}=2$ & buy asset 1 & buy asset 2 & buy asset 2
\end{tabular}

Table 1: Momentum-reversal strategy implemented at time $t$ with short-selling constraint.

returns, one can implement the profitable portfolio strategy of Table 1. Calling $R_{t+1}$ the expected excess return of such strategy at $t+1$, one has

$$
\begin{aligned}
& \mathrm{E}\left[R_{t+1} \mid p_{t}, s_{t}\right]= \\
& = \begin{cases}\frac{1-\delta}{\delta} \frac{\left(1-p_{t}\right)\left(1 / 2+\lambda-p_{t}\right)}{p_{t}\left(1-p_{t}\right)-\delta\left(\pi^{1}-p_{t}\right)\left(p_{t}-\pi^{2}\right)}>0 & \text { if } p_{t}<1 / 2+\lambda \text { and } s_{t}=1, \\
\frac{1-\delta}{\delta} \frac{\left(1-p_{t}\right)\left(1 / 2+\lambda-p_{t}\right)}{p_{t}\left(1-p_{t}\right)-\delta\left(\pi^{1}-p_{t}\right)\left(p_{t}-\pi^{2}\right)}>0 & \text { if } p_{t}<1 / 2-\lambda \text { and } s_{t}=2, \\
\frac{1-\delta}{\delta} \frac{p_{t}\left(p_{t}-1 / 2-\lambda\right)}{p_{t}\left(1-p_{t}\right)-\delta\left(\pi^{1}-p_{t}\right)\left(p_{t}-\pi^{2}\right)}>0 & \text { if } p_{t}>1 / 2+\lambda \text { and } s_{t}=1, \\
\frac{1-\delta}{\delta} \frac{p_{t}\left(p_{t}-1 / 2+\lambda\right)}{p_{t}\left(1-p_{t}\right)-\delta\left(\pi^{1}-p_{t}\right)\left(p_{t}-\pi^{2}\right)}>0 & \text { if } p_{t}>1 / 2-\lambda \text { and } s_{t}=2 .\end{cases}
\end{aligned}
$$

Notice that, exploiting both short-term momentum and long-term reversal, the portfolio has positive expected return between period $t$ and period $t+1$ for every $\sigma$ without resorting to short selling. When $1 / 2-\lambda<p_{t}<1 / 2+\lambda$ the portfolio exploits short-term momentum: given the sign of the last return (implied by the state realized at $t$ ) it goes long in the asset whose news are positive. When, instead, the price goes outside the interval $(1 / 2-\lambda, 1 / 2+\lambda)$, the portfolio exploits long-run reversal: it buys the asset that is underpriced and has positive expected return, i.e. when $p_{t}$ goes below $1 / 2-\lambda$ it buys asset 1 while it buys asset 2 when $p_{t}$ goes above $1 / 2+\lambda$.

By providing a positive expected next period return for every round of trading, such strategy outperforms both the Winners and the Losers portfolio described in the previous section. Figure 6 compares the expected cumulated excess returns after $h$ rounds of trading of strategies implemented in any $\tau=t, t+1, \ldots, t+h-1$ : $A 1$ buys only asset 1, $A 2$ buys only asset 2 , and $2 A M R$ is the portfolio strategy in Table 1. For any level of $p_{t}$ and for any $s_{t}$, the expected cumulated excess return of $2 A M R$ is larger than or equal to the ones entailed by $A 1$ or $A 2$. This implies that $2 A M R$ does better than any possible one-asset Winner or Loser portfolio. 


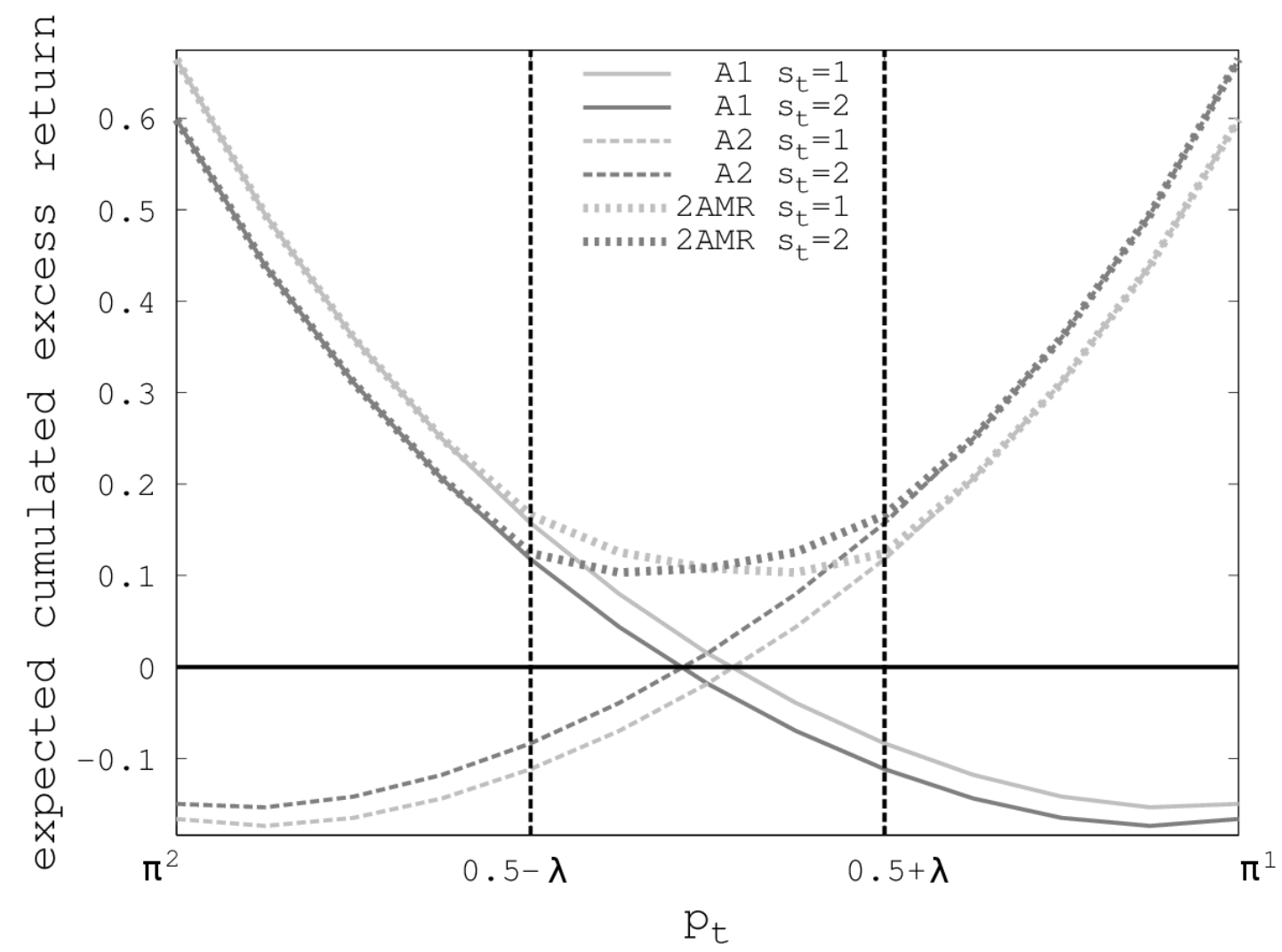

Figure 6: Comparison of expected cumulated excess returns after $h$ rounds of trading of a strategy that buys only asset $1(A 1)$ and of the portfolio strategy in table 1 ( $2 A M R)$. Values of parameters used in the picture: $\delta=0.95, \lambda=0.1$, $\pi^{1}=0.8, \pi^{2}=0.2, h=8$.

\subsection{Zero-cost Momentum-Reversal Strategy}

Having two risky assets in our economy and allowing for short-selling, one can design zero-cost trading strategies with positive pay-off in expectation. These portfolios are not arbitrages in that pay-offs might be positive or negative, but could be considered as statistical arbitrages: if the same trade is repeated over and over again, then, by the law of large numbers, the realized profit is positive even if the strategy has zero cost.13

Indeed, inspired by the strategy found in Section 6.2, consider the portfolio

\footnotetext{
${ }^{13}$ Despite agent demand is not directly derived by an inter-temporal utility maximization, no arbitrages are possible in our model, see also Bottazzi et al. (2017).
} 


\begin{tabular}{c|c|c|c} 
& $p_{t}<1 / 2-\lambda$ & $1 / 2-\lambda<p_{t}<1 / 2+\lambda$ & $p_{t}>1 / 2+\lambda$ \\
\hline$s_{t}=1$ & buy $1 / P_{1, t}$ of asset 1 & buy $1 / P_{1, t}$ of asset 1 & sell $1 / P_{1, t}$ of asset 1 \\
& sell $1 / P_{2, t}$ of asset 2 & sell $1 / P_{2, t}$ of asset 2 & buy $1 / P_{2, t}$ of asset 2 \\
\hline$s_{t}=2$ & buy $1 / P_{1, t}$ of asset 1 & sell $1 / P_{1, t}$ of asset 1 & sell $1 / P_{1, t}$ of asset 1 \\
sell $1 / P_{2, t}$ of asset 2 & buy $1 / P_{2, t}$ of asset 2 & buy $1 / P_{2, t}$ of asset 2
\end{tabular}

Table 2: Zero-cost momentum-reversal strategy implemented at time $t$.

strategy in table 2, Calling $R_{t+1}^{\prime}$ its payoff, one has

$$
\begin{aligned}
& \mathrm{E}\left[R_{t+1}^{\prime} \mid p_{t}, s_{t}\right]= \\
& = \begin{cases}\frac{1-\delta}{\delta} \frac{1 / 2+\lambda-p_{t}}{p_{t}\left(1-p_{t}\right)-\delta\left(\pi^{1}-p_{t}\right)\left(p_{t}-\pi^{2}\right)}>0 & \text { if } p_{t}<1 / 2+\lambda \text { and } s_{t}=1, \\
\frac{1-\delta}{\delta} \frac{1 / 2-\lambda-p_{t}}{p_{t}\left(1-p_{t}\right)-\delta\left(\pi^{1}-p_{t}\right)\left(p_{t}-\pi^{2}\right)}>0 & \text { if } p_{t}<1 / 2-\lambda \text { and } s_{t}=2, \\
\frac{1-\delta}{\delta} \frac{p_{t}-1 / 2-\lambda}{p_{t}\left(1-p_{t}\right)-\delta\left(\pi^{1}-p_{t}\right)\left(p_{t}-\pi^{2}\right)}>0 & \text { if } p_{t}>1 / 2+\lambda \text { and } s_{t}=1, \\
\frac{1-\delta}{\delta} \frac{p_{t}-1 / 2+\lambda}{p_{t}\left(1-p_{t}\right)-\delta\left(\pi^{1}-p_{t}\right)\left(p_{t}-\pi^{2}\right)}>0 & \text { if } p_{t}>1 / 2-\lambda \text { and } s_{t}=2 .\end{cases}
\end{aligned}
$$

Such zero-cost portfolio amplifies the good performance of the strategy in table 1 exploiting short-selling. The intuition that underlies such result is again the presence of momentum and reversal in the market, together with the price bounds $\{1 / 2-\lambda, 1 / 2+\lambda\}$, and the fact that in our economy the two assets have anticorrelated returns and prices. When $p_{t}<1 / 2-\lambda$ or $p_{t}>1 / 2+\lambda$, one asset is overpriced while the other is underpriced, hence one can get a positive expected payoff with zero cost selling the overpriced asset and buying the underpriced one. In this way the strategy is suggesting a Losers-minus-Winners portfolio which exploits reversal. When assets are neither overpriced nor underpriced, $1 / 2-\lambda<$ $p_{t}<1 / 2+\lambda$, then it relies on the information provided by the last realized state of the word in order to build a Winners-minus-Losers portfolio which performs well because of momentum.

\subsection{Dispersion in Beliefs and Dividend Autocorrelation}

Having computed expected returns as a function of the economy parameters, we can analyze what happens to short-term momentum and long-term reversal when these parameters change. Let us start considering what happens when beliefs 

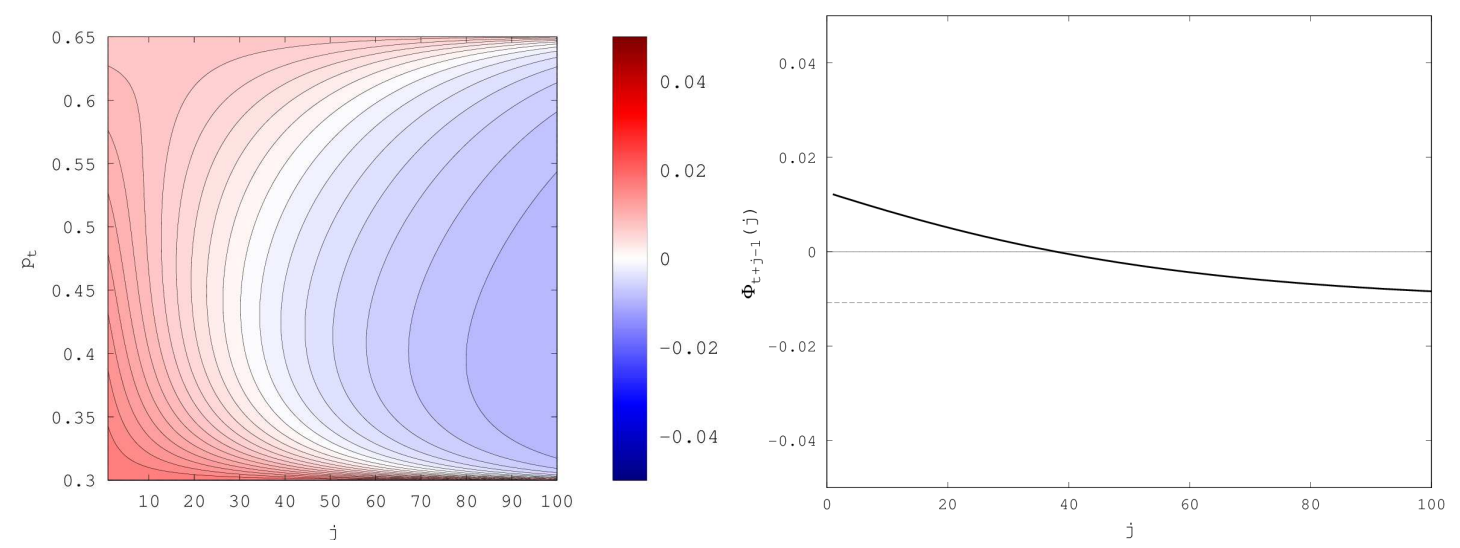

Figure 7: First panel: $\phi_{t+j-1}\left(j, p_{t}\right)$. Second panel: $\Phi_{t+j-1}(j)$. The dashed line represents $\Phi_{\infty}$.

are less dispersed. In Figure 7, we take the same parametrization considered in Figure 4 but modify agents' beliefs to $\pi^{1}=0.65$ and $\pi^{2}=0.3$. Both momentum and reversal decrease in magnitude. The result is general. For $j=1$ one has

$$
\frac{\partial \phi_{t}\left(1, p_{t}\right)}{\partial \pi^{1}}>0 \text { and } \quad \frac{\partial \phi_{t}\left(1, p_{t}\right)}{\partial \pi^{2}}<0
$$

If an agent beliefs become more extreme, then the short-term momentum increases. For the long-run one gets

$$
\frac{\partial \phi_{\infty}\left(p_{t}\right)}{\partial \pi^{1}}<0 \text { and } \frac{\partial \phi_{\infty}\left(p_{t}\right)}{\partial \pi^{2}}>0
$$

show that also long-term reversal gets stronger when agents' disagreement increases.

Some contributions have explored the relation between beliefs dispersion and both momentum and reversal. According to Hong and Stein (1999) "it seems plausible that information about small firms gets out slowly" and the same should hold for analyst coverage, thus one can think that stocks related to small firms and/or to firms with low coverage should induce agents to end up with beliefs that are more dispersed than with large firms and/or high analyst coverage. Under this assumption the empirical results of Hong et al. (2000) and Daniel and Titman (1999) agree with the analysis presented here. Verardo (2009) considers explicitly the impact of beliefs dispersion on momentum. She finds a positive link between beliefs dispersion and momentum profits, thus confirming our finding for what concerns short-term momentum.

The analysis just performed concerning beliefs can be repeated in terms of the autocorrelation coefficient $\lambda$. In Figure 8, we take the same parametrization used 

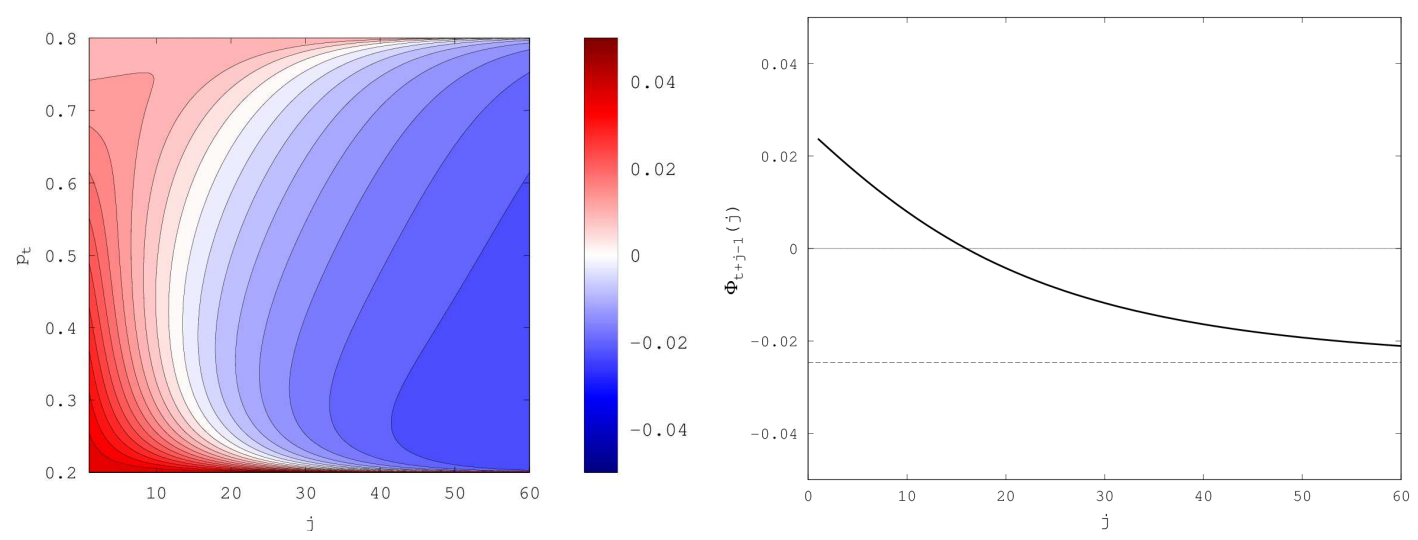

Figure 8: First panel: $\phi_{t+j-1}\left(j, p_{t}\right)$. Second panel: $\Phi_{t+j-1}(j)$. The dashed line represents $\Phi_{\infty}$.

in Figure 4 but increase the value of $\lambda$ to 0.15 . Both a stronger momentum in the short-run and weaker reversal in the long-run emerge. The result is quite general, indeed one has

$$
\frac{\partial \phi_{t}\left(1, p_{t}\right)}{\partial \lambda}>0 \text { and } \frac{\partial \phi_{\infty}\left(p_{t}\right)}{\partial \lambda}>0 .
$$

Thus, when the probability of having the same state of the current period becomes higher, one has larger short-term momentum and (because of its negative sign) a lower long-term reversal. About this point there are not, up to our knowledge, contributions that highlight such link either in theoretical or empirical terms. We leave this investigation for future research.

\section{Conclusions}

In this paper we show that, when financial returns are the result of persistent speculative exchanges due to beliefs heterogeneity, two well-known empirical facts are recovered: short-run momentum and long-run reversal. In our model the source of the two phenomena can be explained considering the following three ingredients: the positive autocorrelation of the dividend process, agents' relative wealth dynamics, and agents' diverse beliefs. The basic mechanism that underlies the relative wealth dynamics is that when good states related to an asset arrive, i.e. the asset pays a dividend, the agent who is investing the most in that asset increases her wealth, generating a positive price variation and thus a positive excess return. In the case of bad states the opposite occurs. This, together with the positive autocorrelation of the dividend process, implies that the probability of observing a variation of agents wealth with a given sign after a variation of the same sign 
is higher than a variation of opposite sign. The result is short-run momentum. However, when we consider long series of states of the same type, the fact that agents invest based on non correct and heterogeneous beliefs generates extreme mispricing. In particular, the overpriced asset has negative expected returns while the underpriced asset has positive expected returns, leading to long-term reversal. The process never settles because under mispricing it is the poorest agent who is favored: she is investing a large fraction of her wealth in the underpriced asset and a small fraction in the overpriced asset and thus, provided her portfolio is diverse enough, she is able to grow more in expectation. This implies that the price eventually reverts back to the mean and returns exhibit long-run reversal. Thus, in our model, the occurrence of momentum and reversal is not linked to investors' under- and over- reaction as in the behavioral finance literature: our analysis unveils a complementary source for the two effects.

Such source proves effective in reproducing the superior performance of a Winners portfolio with respect to a Losers portfolio over short lags and of a Losers portfolio with respect to a Winners portfolio over distant lags. Improving upon these strategies, we also show how to use momentum and reversal price bounds to build strategies that are profitable, in expectation, in every period, both when a short-selling constraint holds and when it does not. Finally, our analysis implies that dividend autocorrelation is positively related to momentum and negatively related to reversal whereas diversity of beliefs is positively related to both momentum and reversal.

\section{References}

Asness, C. S., T. J. Moskowitz, and L. H. Pedersen (2013). Value and momentum everywhere. The Journal of Finance 68(3), 929-985.

Balvers, R., Y. Wu, and E. Gilliland (2000). Mean reversion across national stock markets and parametric contrarian investment strategies. The Journal of Finance 55(2), 745-772.

Barberis, N., A. Shleifer, and R. Vishny (1998). A model of investor sentiment. Journal of Financial Economics 49(3), 307-343.

Beker, P. F. and S. Chattopadhyay (2010). Consumption dynamics in general equilibrium: A characterisation when markets are incomplete. Journal of Economic Theory 145, 2133-2185.

Beker, P. F. and E. Espino (2011). The dynamics of efficient asset trading with heterogeneous beliefs. Journal of Economic Theory 146, 189-229. 
Beker, P. F. and E. Espino (2015). Short-term momentum and long-term reversal of returns under limited enforceability and belief heterogeneity. Technical Report 1096, University of Warwick, Department of Economics.

Bernard, V. L. (1992). Stock price reactions to earnings announcements: A summary of recent anomalous evidence and possible explanations. In R. Thaler (Ed.), Advances in Behavioral Finance. Russell Sage Foundation.

Bernard, V. L. and J. K. Thomas (1989). Post-earnings-announcement drift: delayed price response or risk premium? Journal of Accounting research, 1-36.

Blume, L. and D. Easley (1992). Evolution and market behavior. Journal of Economic Theory 58, 9-40.

Blume, L. and D. Easley (2006). If you are so smart why aren't you rich? Belief selection in complete and incomplete markets. Econometrica 74, 929-966.

Borovička, J. (2015). Survival and long-run dynamics with heterogeneous beliefs under recursive preferences. Working paper, New York University.

Bottazzi, G. and P. Dindo (2015, September). Drift criteria for persistence of discrete stochastic processes on the line. LEM Papers Series 2015/26, Laboratory of Economics and Management (LEM), Sant'Anna School of Advanced Studies, Pisa, Italy.

Bottazzi, G., P. Dindo, and D. Giachini (2017). Long-run heterogeneity in an exchange economy with fixed-mix traders. Economic Theory, forthcoming.

Bottazzi, G. and D. Giachini (2016). Far from the madding crowd: Collective wisdom in prediction markets. LEM Papers Series 2016/14, Laboratory of Economics and Management (LEM), Sant'Anna School of Advanced Studies, Pisa, Italy.

Bottazzi, G. and D. Giachini (2017). Wealth and price distribution by diffusive approximation in a repeated prediction market. Physica A: Statistical Mechanics and its Applications 471, 473-479.

Breiman, L. (1961). Optimal gambling systems for favorable games. Proceedings of the 4th Berkley Symposium on Mathematical Statistics and Probability 1, 63-68.

Cao, D. (2017). Speculation and financial wealth distribution under belief heterogeneity. The Economic Journal, forthcoming.

Chan, L. K., N. Jegadeesh, and J. Lakonishok (1996). Momentum strategies. The Journal of Finance 51(5), 1681-1713. 
Chopra, N., J. Lakonishok, and J. R. Ritter (1992). Measuring abnormal performance: do stocks overreact? Journal of financial Economics 31(2), 235-268.

Cogley, T., T. Sargent, and V. Tsyrennikov (2013). Wealth dynamics in a bond economy with heterogeneous beliefs. The Economic Journal 124, 1-30.

Condie, S. (2008). Living with ambiguity: prices and survival when investors have heterogeneous preferences for ambiguity. Economic Theory 36, 81-108.

Coury, T. and E. Sciubba (2012). Belief heterogeneity and survival in incomplete markets. Economic Theory 49, 37-58.

Cutler, D. M., J. M. Poterba, and L. H. Summers (1991). Speculative dynamics. The Review of Economic Studies 58(3), 529-546.

Cvitanić, J., E. Jouini, S. Malamud, and C. Napp (2012). Financial markets equilibrium with heterogeneous agents. Review of Finance 16(1), 285-321.

Daniel, K., D. Hirshleifer, and A. Subrahmanyam (1998). Investor psychology and security market under-and overreactions. The Journal of Finance 53(6), $1839-1885$.

Daniel, K. and S. Titman (1999). Market efficiency in an irrational world. Financial Analysts Journal 55(6), 28-40.

De Bondt, W. F. and R. Thaler (1985). Does the stock market overreact? The Journal of Finance 40(3), 793-805.

De Bondt, W. F. and R. Thaler (1987). Further evidence on investor overreaction and stock market seasonality. The Journal of Finance 42(3), 557-581.

Dindo, P. (2015). Survival in speculative markets. LEM Working Paper 2015-32, Scuola Superiore Sant'Anna, Pisa.

Dindo, P. and F. Massari (2017). The wisdom of the crowd in dynamic economies. Technical report, Department of Economics, University of Venice "Ca'Foscari".

Evstigneev, I., T. Hens, and K. Schenk-Hoppé (2008). Globally evolutionary stable portfolio rules. Journal of Economic Theory 140, 197-228.

Friedman, M. (1953). Essays in Positive Economics. Univ. Chicago Press.

Gigerenzer, G. and H. Brighton (2009). Homo heuristicus: Why biased minds make better inferences. Topics in Cognitive Science 1(1), 107-143. 
Gigerenzer, G. and W. Gaissmaier (2011). Heuristic decision making. Annual review of psychology 62, 451-482.

Gigerenzer, G., P. M. Todd, and A. R. Group (1999). Simple heuristics that make us smart. Oxford University Press.

Gropp, J. (2004). Mean reversion of industry stock returns in the us, 1926-1998. Journal of Empirical Finance 11(4), 537-551.

Guerdjikova, A. and E. Sciubba (2015). Survival with ambiguity. Journal of Economic Theory 155, 50-94.

Hong, H., T. Lim, and J. C. Stein (2000). Bad news travels slowly: Size, analyst coverage, and the profitability of momentum strategies. The Journal of Finance 55(1), 265-295.

Hong, H. and J. C. Stein (1999). A unified theory of underreaction, momentum trading, and overreaction in asset markets. The Journal of Finance 54 (6), 21432184.

Hong, H. and J. C. Stein (2007). Disagreement and the stock market (digest summary). Journal of Economic perspectives 21(2), 109-128.

Jegadeesh, N. (1990). Evidence of predictable behavior of security returns. Journal of Finance, 881-898.

Jegadeesh, N. and S. Titman (1993). Returns to buying winners and selling losers: Implications for stock market efficiency. The Journal of Finance 48(1), 65-91.

Jegadeesh, N. and S. Titman (2001). Profitability of momentum strategies: An evaluation of alternative explanations. The Journal of Finance 56(2), 699-720.

Jouini, E. and C. Napp (2006). Heterogeneous beliefs and asset pricing in discrete time: An analysis of pessimism and doubt. Journal of Economic Dynamics and Control 30(7), 1233-1260.

Jouini, E. and C. Napp (2007). Consensus consumer and intertemporal asset pricing with heterogeneous beliefs. The Review of Economic Studies 74(4), 1149-1174.

Kelly, J. (1956). A new interpretation of information rates. Bell System Technical Journal 35, 917-926.

Kets, W., D. M. Pennock, R. Sethi, and N. Shah (2014). Betting strategies, market selection, and the wisdom of crowds. In Twenty-Eighth AAAI Conference on Artificial Intelligence. 
Lewellen, J. (2002). Momentum and autocorrelation in stock returns. The Review of Financial Studies 15(2), 533-564.

Lo, A. W. and A. C. MacKinlay (1990). When are contrarian profits due to stock market overreaction? The Review of Financial Studies 3(2), 175-205.

Moskowitz, T. J. and M. Grinblatt (1999). Do industries explain momentum? The Journal of Finance 54 (4), 1249-1290.

Moskowitz, T. J., Y. H. Ooi, and L. H. Pedersen (2012). Time series momentum. Journal of Financial Economics 104(2), 228-250.

Mukherji, S. (2011). Are stock returns still mean-reverting? Review of Financial Economics 20(1), 22-27.

Poterba, J. and L. Summers (1988). The persistence of volatility and stock market fluctuations. American Economic Review 76 (5), 1142-1151.

Rouwenhorst, K. G. (1998). International momentum strategies. The Journal of Finance 53(1), 267-284.

Sandroni, A. (2000). Do markets favor agents able to make accurate predictions. Econometrica 68(6), 1303-1341.

Sandroni, A. (2005). Efficient markets and bayes rule. Economic Theory 26(4), 741-764.

Sciubba, E. (2005). Asymmetric information and survival in financial markets. Economic Theory 25, 353-379.

Verardo, M. (2009). Heterogeneous beliefs and momentum profits. Journal of Financial and Quantitative Analysis 44(04), 795-822.

Yan, H. (2008). Natural selection in financial markets: Does it work? Management Science 54, 1935-1950.

\section{A Proof of Proposition 4.1}

Since $0<\pi^{2}<\pi^{1}<1$, there exists a $\varepsilon>0$ such that $\varepsilon \leq \pi^{2}<\pi^{1} \leq 1-\varepsilon$. Let

$$
z_{t}=\log \frac{w_{t}}{1-w_{t}}=\log \frac{p_{t}-\pi^{2}}{\pi^{1}-p_{t}}
$$


From (12), given that $p \in\left[\pi^{2}, \pi^{1}\right]$, it is easy to show that for $s_{t+1}=1$ it is

$$
0<z_{t+1}-z_{t}<\log \frac{1-\varepsilon}{\varepsilon}
$$

and for $s_{t+1}=2$ it is

$$
\log \frac{\varepsilon}{1-\varepsilon}<z_{t+1}-z_{t}<0 .
$$

Since $z_{t+1}-z_{t}$ is continuous in $p$, these inequalities imply that $\left\{z_{t}\right\}$ is a bounded increments process with finite positive and negative increments as defined in Bottazzi and Dindo (2015). With $i, j \in\{1,2\}$ and $i \neq j$ define the two quantities

$$
A_{j}^{+}=\left(\frac{1}{2}+\lambda\right) \log \left(\frac{\delta \pi^{j}+(1-\delta) \pi^{i}}{\pi^{j}}\right)+\left(\frac{1}{2}-\lambda\right) \log \left(\frac{1-\delta \pi^{j}-(1-\delta) \pi^{i}}{1-\pi^{j}}\right)
$$

and

$$
A_{j}^{-}=\left(\frac{1}{2}-\lambda\right) \log \left(\frac{\delta \pi^{j}+(1-\delta) \pi^{i}}{\pi^{j}}\right)+\left(\frac{1}{2}+\lambda\right) \log \left(\frac{1-\delta \pi^{j}-(1-\delta) \pi^{i}}{1-\pi^{j}}\right) .
$$

Call $\mu(z, s)=\mathrm{E}\left[z_{t+1} \mid z_{t}=z, s_{t}=s\right]-z$ and notice that $\lim _{z \rightarrow+\infty} \mu(z, 1)=-A_{1}^{+}$, $\lim _{z \rightarrow+\infty} \mu(z, 2)=-A_{1}^{-}, \lim _{z \rightarrow-\infty} \mu(z, 1)=A_{2}^{+}$and $\lim _{z \rightarrow-\infty} \mu(z, 1)=A_{2}^{-}$. Thus, if the conditions stated in the first part of the Proposition are satisfied, Theorem 2.2 of Bottazzi and Dindo (2015) applies, the process $\left\{z_{t}\right\}$ is persistent and both agents survive. The last statement of the Proposition about the limits of $p_{t}$ follows from the finite positive and negative increments of $\left\{z_{t}\right\}$.

\section{B Proof of Corollary 4.1}

Conditions (13) and (14) can be written as

$$
\begin{aligned}
& \rho\left(\frac{1}{2}+\lambda, \delta \pi^{j}+(1-\delta) \pi^{i}, \pi^{j}\right)>0, \\
& \rho\left(\frac{1}{2}-\lambda, \delta \pi^{j}+(1-\delta) \pi^{i}, \pi^{j}\right)>0,
\end{aligned}
$$

with

$$
\rho\left(\pi, \pi^{\prime}, \pi^{\prime \prime}\right)=\pi \log \frac{\pi^{\prime}}{\pi^{\prime \prime}}+(1-\pi) \log \frac{1-\pi^{\prime}}{1-\pi^{\prime \prime}} .
$$

By Jensen's inequality, it is

$$
\log \left(1+\frac{\left(\pi^{\prime \prime}-\pi^{\prime}\right)\left(\pi^{\prime \prime}-\pi\right)}{\pi^{\prime \prime}\left(1-\pi^{\prime \prime}\right)}\right)>\rho\left(\pi, \pi^{\prime}, \pi^{\prime \prime}\right) .
$$


Thus, if the right hand side is positive, the argument of the logarithm in the left hand side must be greater than one. Substituting the values for $\pi, \pi^{\prime}$ and $\pi^{\prime \prime}$ and remembering that $\delta \in(0,1)$, this implies

$$
\left(\pi^{j}-\pi^{i}\right)\left(\pi^{j}-\frac{1}{2}-\lambda\right)>0 \quad \text { and } \quad\left(\pi^{j}-\pi^{i}\right)\left(\pi^{j}-\frac{1}{2}+\lambda\right)>0
$$

which, remembering that $0<\pi^{1}<\pi^{2}<1$, is equivalent to the assertion.

\section{Proof of Propositions 5.2}

From the price process $(12)$ and Lemma 5.1 we have

$$
\begin{aligned}
\mathrm{E}\left[r_{1, t+1} \mid \mathcal{R}_{t}^{+}(1), p_{t}\right] & =\frac{1-\delta}{\delta} \frac{\left(1-p_{t}\right)\left(1 / 2+\lambda-p_{t}\right)}{p_{t}\left(1-p_{t}\right)-\delta\left(\pi^{1}-p_{t}\right)\left(p_{t}-\pi^{2}\right)} \\
\mathrm{E}\left[r_{1, t+1} \mid \mathcal{R}_{t}^{-}(1), p_{t}\right] & =\frac{1-\delta}{\delta} \frac{\left(1-p_{t}\right)\left(1 / 2-\lambda-p_{t}\right)}{p_{t}\left(1-p_{t}\right)-\delta\left(\pi^{1}-p_{t}\right)\left(p_{t}-\pi^{2}\right)}
\end{aligned}
$$

Averaging with respect the the invariant distribution of the states on the world $\pi^{*}$ gives

$$
\phi_{t}\left(1, p_{t}\right)=\frac{1}{2} \mathrm{E}\left[r_{1, t+1} \mid \mathcal{R}_{t}^{+}(1), p_{t}\right]-\frac{1}{2} \mathrm{E}\left[r_{1, t+1} \mid \mathcal{R}_{t}^{-}(1), p_{t}\right]
$$

which gives the required expression. The fact that $\phi_{t}\left(1, p_{t}\right)>0$ for all $(t, \sigma)$ and for all $p_{t} \in\left(\pi^{2}, \pi^{1}\right)$ implies $\Phi_{t}(1)>0$ for all $(t, \sigma)$, irrespective of the price distribution.

\section{Proof of Propositions 5.3}

From (15)-16) in the proof above and Lemma 5.1, irrespective of the value of $p_{t}$ one has

$$
\begin{aligned}
& \lim _{j \rightarrow \infty} \mathrm{E}\left[r_{1, t+j} \mid \mathcal{R}_{t+j-1}^{+}(j), p_{t}\right]=\mathrm{E}\left[r_{1, t+1} \mid \mathcal{R}_{t}^{+}(1), \pi^{1}\right]=\frac{1-\delta}{\delta} \frac{1 / 2+\lambda-\pi^{1}}{\pi^{1}}, \\
& \lim _{j \rightarrow \infty} \mathrm{E}\left[r_{1, t+j} \mid \mathcal{R}_{t+j-1}^{-}(j), p_{t}\right]=\mathrm{E}\left[r_{1, t+1} \mid \mathcal{R}_{t}^{-}(1), \pi^{2}\right]=\frac{1-\delta}{\delta} \frac{1 / 2-\lambda-\pi^{2}}{\pi^{2}} .
\end{aligned}
$$

Averaging with respect the the invariant distribution of the states on the world $\pi^{*}$ gives

$$
\phi_{\infty}\left(p_{t}\right)=\frac{1}{2} \mathrm{E}\left[r_{1, t+1} \mid \mathcal{R}_{t}^{+}(1), \pi^{1}\right]-\frac{1}{2} \mathrm{E}\left[r_{1, t+1} \mid \mathcal{R}_{t}^{-}(1), \pi^{2}\right],
$$

which gives the required expression. Notice that this expression does not depend on $p_{t}$, hence $\Phi_{\infty}=\phi_{\infty}\left(p_{t}\right)$. 